\title{
Stochastic modeling of the time variability of ALMA calibrators
}

\author{
A. E. Guzmán, ${ }^{1}$ C. Verdugo,${ }^{2}$ H. Nagai, ${ }^{1}$ Y. Contreras,${ }^{3}$ G. \\ Marinello, ${ }^{2}$ R. Kneissl, ${ }^{2}$ K. Nakanishi, ${ }^{1}$ J. Ueda. ${ }^{1}$ \\ ${ }^{1}$ National Astronomical Observatory of Japan, 2-21-1 Osawa, Mitaka, Tokyo \\ 181-8588, Japan \\ 2 Joint Alma Observatory (JAO), Alonso de Córdova 3107, Vitacura, Santiago, Chile \\ ${ }^{3}$ Leiden Observatory, Leiden University, PO Box 9513, NL-2300 RA Leiden, the \\ Netherlands \\ 12 August 2019 \\ E-mail: andres.guzman@nao.ac.jp
}

\begin{abstract}
Characterizing the variability of the extragalactic sources used for calibration in the Atacama Large Millimeter/Sub-millimeter Array (ALMA) is key to assess the flux scale uncertainty of science observations. To this end, we model the variability of 39 quasars which have been used by ALMA as secondary flux calibrators using continuous time stochastic processes. This formalism is specially adapted to the multi-frequency, quasi-periodic sampling which characterizes the calibration monitoring of ALMA. We find that simple mixtures of Ornstein-Uhlenbeck processes can describe well the flux and spectral index variability of these sources for Bands 3 and 7 (91.5 and 103.5, and $343.5 \mathrm{GHz}$, respectively). The spectral shape of the calibrators are characterized by negative spectral indices, mostly between -0.35 and -0.80 , and with additional concavity. The model provides forecasts, interpolations, and uncertainty estimations for the observed fluxes that depend on the intrinsic variability of the source. These can be of practical use for the ALMA data calibrator survey and data quality assurance.
\end{abstract}

Keywords: methods: statistical — methods: data analysis — submillimeter: galaxies — radio continuum: galaxies

Submitted to: Publications of the Astronomical Society of the Pacific 


\section{Introduction}

The flux density scale and calibration of the science observations performed by the Atacama Large Millimeter/Sub-millimeter Array (ALMA) relies heavily on contemporaneous comparison with calibrator sources. Properties of these calibrators need to be predictable to an acceptable uncertainty level. The flux scaling, in particular, depends ultimately on comparison with solar system objects ( $\mathrm{SSO}$ ) which are used as primary flux calibrators. For these, (sub-)millimeter emission models are accurate within a 3-5\% level (Butler 2012).

In the ideal case, each observation should include one measurement of a primary flux calibrator, whose flux is then bootstrapped to an amplitude calibrator (Moreno \& Guilloteau 2002). The scarcity of SSO calibrators and its restricted location near the ecliptic forces us to rely on bright quasars for flux calibration in case SSO observations are inconvenient or inaccessible (Fomalont et al. 2014). Quasars have some advantages as calibrators for ALMA compared with SSO: they are in most cases unresolved pointlike sources with little spectral features like emission or absorption lines, which makes them ideal for phase and bandpass calibration.

However, quasars are also known to be highly variable. Moreover, most ALMA calibrators are bright $\mathrm{mm} / \mathrm{sub}$-mm blazars of various types (Bonato et al. 2018). Blazars are known to be amongst the most variable type of quasar (Falomo et al. 2014, Ulrich et al. 1997, Wagner \& Witzel 1995). In contrast to the more predictable variability observed toward SSOs, the complexity of the quasar phenomena makes it very difficult to accurately predict its flux variations. Therefore, their use as secondary flux calibrators requires constant monitoring and cross calibration against primary flux calibrators. To this end, the ALMA calibration procedure relies on frequent observations of a subset of $\sim 40$ bright and homogeneously distributed in the sky extragalactic sources known as the "grid" calibrators. These are monitored and compared with near-to-simultaneous observations of SSO objects every 10-14 days (van Kempen et al. 2012, Warmels et al. 2018). The typical calibration procedure for ALMA therefore includes an observation of a grid source whose flux is assumed to be known. This assumed flux is calculated by interpolating the flux and spectral index from the closest measurements calibrated against primary flux calibrators. This same grid source is commonly used to determine the bandpass response as well. The amplitude and phase calibrator is usually another, fainter quasar, located close $\left(\leq 10^{\circ}\right.$ for $\mathrm{mm}$ wavelength and compact array configuration; and $\leq 2^{\circ}$ if possible for sub-mm wavelength and extended configurations) to the science target, whose flux is determined from comparison with the flux calibrator and transferred to the science target.

In consequence, the flux density of the grid quasar used as secondary flux calibrator has an additional uncertainty due to its intrinsic variability, uncertainty that is transmitted to any source which uses said quasar for flux scaling. This uncertainty depends on how much time has elapsed between the closest comparison performed against a primary flux calibrator and the time of observation: the larger 
this time interval, the larger the uncertainty. Thus, it is key to adopt strategies to minimize and quantify the uncertainties produced by the time variability of the secondary flux calibrator. Intrinsic, non-predictable variability of the flux calibrator has not been traditionally included explicitly as a source of uncertainty before (Yun et al. 1998, Moreno \& Guilloteau 2002, van Kempen et al. 2014).

In order to estimate quantitatively the uncertainty of the flux of the quasars due to variability, in this work we model the calibrator's fluxes using time-series stochastic processes. Stochastic time series (e.g., Scargle 1981, Scargle 1982, Feigelson et al. 2018) have been used in astronomy to model the light-curves of time-varying phenomena which have a random or unpredictable component. This randomness can arise in a variety of forms, either due to an intrinsic unpredictability of the phenomena, or due to uncertainties introduced in the measurement process.

The main objective of this work is to characterize the variability of the ALMA grid calibrators statistically. The formalism is largely based on the Ornstein-Uhlenbeck (OU) mixture models described in Kelly et al. (2009) and Kelly et al. (2011) (and generalized in Kelly et al. 2014), which allows us to derive flux predictions, interpolations, and time dependent uncertainties. Albeit the method may also provide constrains on the actual, physical emission mechanisms of these blazars, this is not the main driving goal of the present study. Quasar variability is thought to originate from, for example, variable accretion onto the central black hole, variable jet ejection, relativistic beaming and amplification, development of instabilities, and from line-of-sight effects like occultation events and stellar microlensing from intervening galaxies. Despite the physical interpretation of the optical variability proposed by Kelly et al. (2009) is unlikely to be applicable to the physics of blazar emission, the techniques and stochastic modeling are well adapted (as we also show in the present work) to observed variability at $\mathrm{mm} / \mathrm{sub}-\mathrm{mm}$ wavelengths.

Section 2 describes briefly the ALMA calibrator public dataset. In Section 3 we present the model and its hypotheses. We find best-fit models for the grid calibrators and describe these results in Section 4. We discuss some applications of the modeling to the calibration and quality assurance process of ALMA in section Section 5. Section 6 briefly summarizes the mains results of this work.

\section{ALMA grid calibrator data}

The list of secondary flux calibrators known as the grid sources is given by Table 10.1 from Warmels et al. (2018). Flux densities, positions, and calibrator type can be obtained from the online source catalogue. $\ddagger$ The goals of the grid monitoring calibration survey are to quantify the variability of calibrators, provide up to date flux densities, and identify the best bandpass candidates and investigate their suitability as secondary flux calibrators. The observational aim for the grid sample is to obtain a minimum of one Band 3 and one Band 6 or 7 measurement every two weeks. Band 3 was selected

$\ddagger$ https://almascience.org/sc/ 
Stochastic variability of ALMA calibrators

Table 1. Observational characteristics of the core grid calibrators list. Columns (1) to (3) show the name of the source, its Right Ascension (J2000), and its Declination (J2000), respectively. Columns (4) and (5) show the first and last day of observation of the light-curves presented in this study. Column (6) shows the total number of measurements. Columns (7) and (8) show the mean flux at the fiducial frequency of $100 \mathrm{GHz}$ and the mean spectral index fitted to the data according to (1), respectively.

Column (9) shows the variability index (Equation (2)).

\begin{tabular}{|c|c|c|c|c|c|c|c|c|c|}
\hline & Source & $\begin{array}{l}\text { RA } \\
\text { (J2000) }\end{array}$ & $\begin{array}{l}\text { DEC } \\
(\text { J2000) }\end{array}$ & Start & End & $\begin{array}{r}\# \\
\text { meas. }\end{array}$ & $\begin{array}{r}\left\langle F_{100}\right\rangle \\
(\mathrm{Jy})\end{array}$ & $\langle\alpha\rangle$ & $\begin{array}{r}v i x \\
(\%)\end{array}$ \\
\hline 1 & J0006-0623 & 00:06:13.893 & $-06: 23: 35.34$ & $2013-07-23$ & 2018-07-04 & 387 & 3.85 & -0.58 & 26.1 \\
\hline 2 & $\mathrm{~J} 0237+2848$ & $02: 37: 52.406$ & $+28: 48: 08.99$ & 2012-06-30 & 2018-07-02 & 366 & 2.13 & -0.62 & 12.8 \\
\hline 3 & $\mathrm{~J} 0238+1636$ & 02:38:38.930 & $+16: 36: 59.27$ & 2012-06-30 & 2018-07-02 & 446 & 1.56 & -0.49 & 33.0 \\
\hline 4 & J0319+4130 & 03:19:48.160 & $+41: 30: 42.11$ & 2012-08-15 & 2018-06-30 & 286 & 18.3 & -0.73 & 16.5 \\
\hline 5 & J0334-4008 & $03: 34: 13.654$ & $-40: 08: 25.40$ & 2012-06-30 & 2018-07-07 & 506 & 0.762 & -0.67 & 43.0 \\
\hline 6 & J0423-0120 & 04:23:15.801 & $-01: 20: 33.07$ & 2012-06-30 & 2018-07-07 & 599 & 1.63 & -0.51 & 60.4 \\
\hline 7 & $\mathrm{~J} 0510+1800$ & 05:10:02.369 & $+18: 00: 41.58$ & 2012-07-18 & 2018-07-07 & 448 & 2.54 & -0.45 & 28.6 \\
\hline 8 & J0519-4546 & 05:19:49.723 & $-45: 46: 43.85$ & 2012-07-18 & 2018-07-07 & 495 & 1.2 & -0.39 & 11.9 \\
\hline 9 & J0522-3627 & 05:22:57.985 & $-36: 27: 30.85$ & 2012-07-18 & 2018-07-07 & 463 & 5.57 & -0.24 & 19.9 \\
\hline 10 & J0538-4405 & 05:38:50.362 & $-44: 05: 08.94$ & 2012-07-31 & 2018-07-07 & 563 & 2.04 & -0.65 & 34.6 \\
\hline 11 & J0635-7516 & $06: 35: 46.508$ & $-75: 16: 16.82$ & 2012-06-30 & 2018-07-07 & 758 & 1.25 & -0.92 & 12.1 \\
\hline 12 & $\mathrm{~J} 0750+1231$ & $07: 50: 52.046$ & $+12: 31: 04.83$ & 2012-10-06 & 2018-07-07 & 373 & 1.32 & -0.67 & 13.9 \\
\hline 13 & J0854+2006 & $08: 54: 48.875$ & $+20: 06: 30.64$ & $2012-08-26$ & 2018-07-07 & 451 & 4.54 & -0.46 & 29.1 \\
\hline 14 & J0904-5735 & 09:04:53.179 & $-57: 35: 05.78$ & 2015-05-31 & 2018-07-07 & 95 & 1.16 & -0.42 & 18.3 \\
\hline 15 & J1037-2934 & $10: 37: 16.080$ & $-29: 34: 02.81$ & 2012-08-26 & 2018-07-07 & 481 & 1.22 & -0.52 & 26.2 \\
\hline 16 & $\mathrm{~J} 1058+0133$ & $10: 58: 29.605$ & $+01: 33: 58.82$ & 2012-10-06 & 2018-07-05 & 389 & 4.26 & -0.48 & 20.1 \\
\hline 17 & $\mathrm{~J} 1107-4449$ & 11:07:08.694 & $-44: 49: 07.62$ & 2012-10-06 & 2018-07-07 & 439 & 1.16 & -0.76 & 11.3 \\
\hline 18 & $\mathrm{~J} 1127-1857$ & 11:27:04.392 & $-18: 57: 17.44$ & 2013-12-29 & 2018-07-07 & 84 & 0.93 & -0.62 & 19.7 \\
\hline 19 & $\mathrm{~J} 1146+3958$ & $11: 46: 58.298$ & $+39: 58: 34.30$ & $2012-08-26$ & 2018-07-05 & 266 & 1.2 & -0.56 & 47.5 \\
\hline 20 & $\mathrm{~J} 1229+0203$ & 12:29:06.700 & $+02: 03: 08.60$ & 2012-06-30 & 2018-07-05 & 493 & 9.42 & -0.79 & 32.7 \\
\hline 21 & $\mathrm{~J} 1256-0547$ & $12: 56: 11.167$ & $-05: 47: 21.53$ & 2012-06-30 & 2018-07-05 & 612 & 13 & -0.58 & 19.0 \\
\hline 22 & $\mathrm{~J} 1331+3030$ & $13: 31: 08.288$ & $+30: 30: 32.96$ & 2012-08-02 & 2018-07-05 & 35 & 0.742 & -1.1 & 4.4 \\
\hline 23 & $\mathrm{~J} 1337-1257$ & $13: 37: 39.783$ & $-12: 57: 24.69$ & 2012-06-30 & 2018-07-05 & 437 & 3.55 & -0.6 & 24.8 \\
\hline 24 & J1427-4206 & $14: 27: 56.298$ & $-42: 06: 19.44$ & 2012-06-30 & 2018-07-05 & 554 & 3.4 & -0.58 & 27.3 \\
\hline 25 & J1517-2422 & $15: 17: 41.813$ & $-24: 22: 19.48$ & 2012-06-30 & 2018-07-05 & 470 & 2.39 & -0.29 & 24.2 \\
\hline 26 & $\mathrm{~J} 1550+0527$ & $15: 50: 35.269$ & $+05: 27: 10.45$ & 2012-06-30 & 2018-07-05 & 538 & 1.01 & -0.72 & 5.0 \\
\hline 27 & $\mathrm{~J} 1617-5848$ & $16: 17: 17.891$ & $-58: 48: 07.86$ & 2012-06-30 & 2018-07-05 & 554 & 1.07 & -0.91 & 15.6 \\
\hline 28 & $\mathrm{~J} 1642+3948$ & $16: 42: 58.810$ & $+39: 48: 36.99$ & 2012-07-29 & 2018-07-04 & 330 & 3.19 & -0.74 & 17.6 \\
\hline 29 & $\mathrm{~J} 1733-1304$ & 17:33:02.706 & $-13: 04: 49.55$ & 2012-06-30 & 2018-07-05 & 527 & 2.84 & -0.67 & 10.9 \\
\hline 30 & $\mathrm{~J} 1751+0939$ & $17: 51: 32.819$ & $+09: 39: 00.73$ & 2012-06-30 & 2018-07-04 & 454 & 2.9 & -0.47 & 30.1 \\
\hline 31 & J1924-2914 & $19: 24: 51.056$ & $-29: 14: 30.12$ & 2012-06-30 & 2018-07-04 & 745 & 5.56 & -0.65 & 10.4 \\
\hline 32 & J2000-1748 & 20:00:57.090 & $-17: 48: 57.67$ & 2013-12-30 & 2018-07-04 & 126 & 1.03 & -0.44 & 41.2 \\
\hline 33 & $\mathrm{~J} 2025+3343$ & $20: 25: 10.842$ & $+33: 43: 00.21$ & 2012-06-30 & 2018-07-02 & 483 & 1.46 & -0.8 & 30.2 \\
\hline 34 & $\mathrm{~J} 2056-4714$ & $20: 56: 16.360$ & $-47: 14: 47.63$ & 2012-06-30 & 2018-07-04 & 568 & 1.22 & -0.63 & 21.7 \\
\hline 35 & $\mathrm{~J} 2148+0657$ & 21:48:05.459 & $+06: 57: 38.60$ & 2012-06-30 & 2018-07-04 & 601 & 2.09 & -0.96 & 23.1 \\
\hline 36 & $\mathrm{~J} 2232+1143$ & $22: 32: 36.409$ & $+11: 43: 50.90$ & 2012-06-30 & 2017-09-29 & 357 & 3.76 & -0.48 & 40.5 \\
\hline 37 & $\mathrm{~J} 2253+1608$ & $22: 53: 57.748$ & $+16: 08: 53.56$ & 2013-07-23 & 2018-07-04 & 350 & 14.8 & -0.53 & 17.4 \\
\hline 38 & $\mathrm{~J} 2258-2758$ & $22: 58: 05.963$ & $-27: 58: 21.26$ & 2012-06-30 & 2018-07-04 & 737 & 1.93 & -0.68 & 56.3 \\
\hline 39 & J2357-5311 & $23: 57: 53.266$ & $-53: 11: 13.69$ & 2012-06-30 & 2018-07-04 & 755 & 0.954 & -0.77 & 15.0 \\
\hline
\end{tabular}




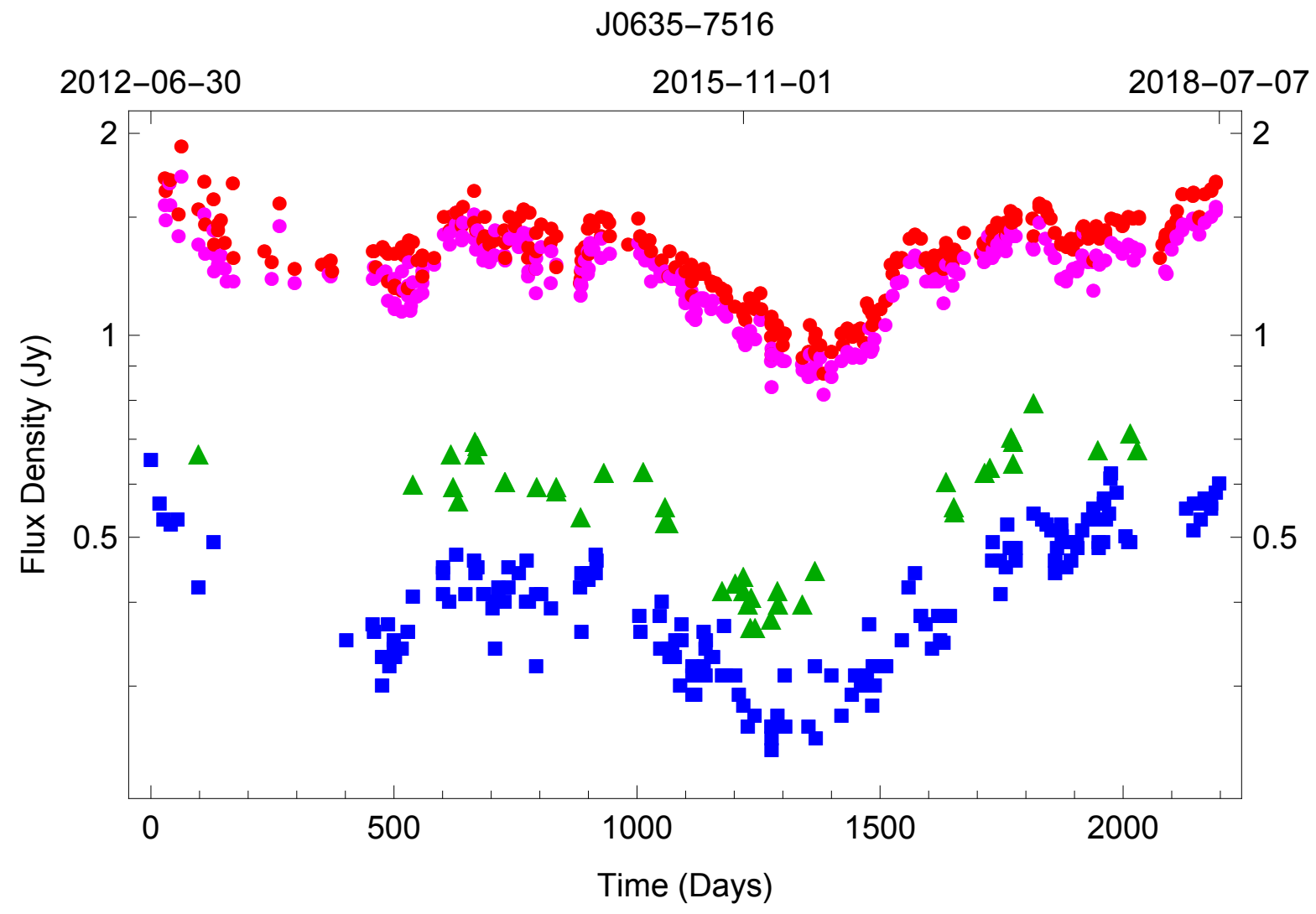

Figure 1. Flux density light-curve for the source with the largest number of measurements, J0635-7516. Band 6, and 7 data are marked with green triangles and blue squares, respectively. Red and magenta circles indicate LSB and USB Band 3 data, respectively.

as the lower frequency because of the smaller weather effects, higher sensitivity, and better instrumental stability. Band 7 was preferred as the second frequency because it combines a large frequency lever arm to estimate the calibrator's spectral index with a relatively large fraction ( $>40 \%$ ) of observing time with compatible weather conditions. Band 6 is the fallback option in case Band 7 observations are not possible, and there are instances in which Band 3 observations are the only option. In some cases, Band 6 data are also taken in addition to the Band 3 and 7 observations. Data reduction and treatment of the calibration data roughly follows the same procedures as normal science observations, but the specific characteristics have been adapted through the years to accommodate the needs and constrains of the observatory. Currently and from Cycle 5 on, observations for the calibrator survey are performed using the Morita ALMA compact array (ACA) and the ACA correlators. The grid calibrators are observed in partially overlapping LST range groups together (ideally) with one of the main primary flux calibrators: Uranus, Neptune, Callisto, Ganymede and Mars. After a pointing calibration, system temperature measurement helps correcting for atmospheric opacity including the most conspicuous atmospheric absorption lines. The bandpass solution is determined from the brightest source of each LST group. Phase self-calibration is 

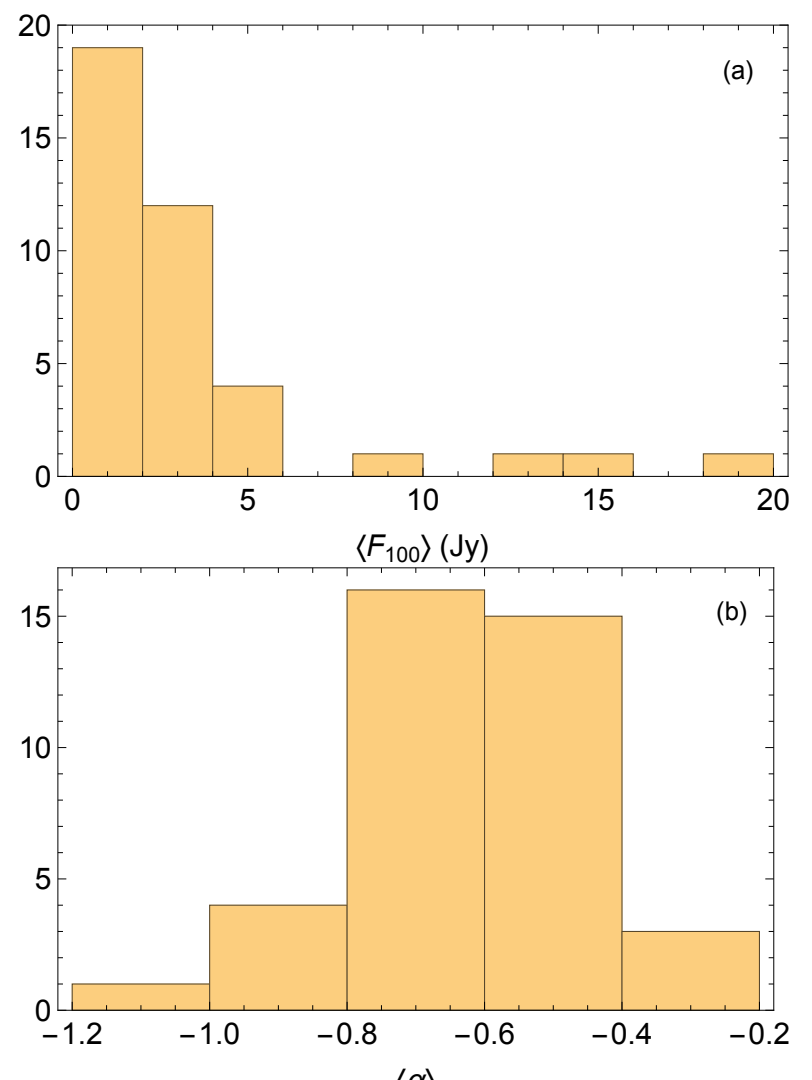

$\langle\alpha\rangle$

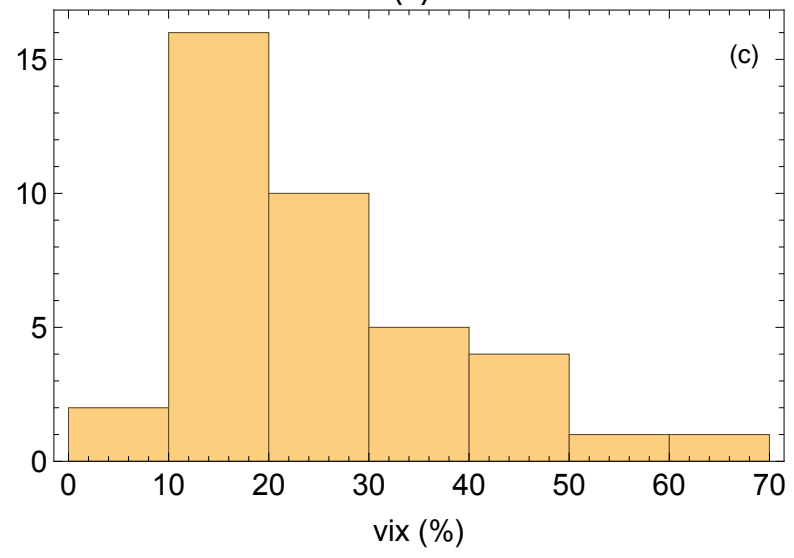

Figure 2. Panels (a) to (c) show, respectively, the histograms of $\left\langle F_{100}\right\rangle,\langle\alpha\rangle$, and vix from Table 1.

performed directly onto the targets. On-source time spent on each grid calibrator varies between 2-4 minutes. More details about the design, observational strategy, and data reduction procedure of the grid calibrator survey can be found in van Kempen et al. (2014).

Table 1 shows some of the observational characteristics of 39 grid calibrator sources. The Joint ALMA Observatory (JAO) revises and updates the list of sources used for secondary flux calibration, which makes the full set of sources being used to slightly vary over time. The sample in Table 1 can be considered the "core" set of sources used by ALMA at least during Cycle 6. Columns (1) to (6) show, respectively, the source 
name, right ascension, declination, the starting day of observations (as it appears in the publicly available catalog), the last day of observations considered in this study, and the number of measured flux densities considered in this study. These sources have been observed approximately every two weeks, but in some cases with significant irregularity. There are four grid calibrators with more sporadic observations which have less than 150 measurements in total, J0904-5735, J1127-1857, J2000-1748, and J1331+3030. These were included as grid calibrators only during the last year. This explains why they have less measurements: the calibrator survey for non-grid sources monitors a sample of approximately 800 quasars (van Kempen et al. 2014), with necessarily a much lower cadence compared to the grid survey.

The bulk $(\geq 90 \%)$ of the grid source observations consist of measurements of the flux densities at Bands 3 and 7, mostly at 91.5 and $103.5 \mathrm{GHz}$, and at $343.5 \mathrm{GHz}$, respectively. Because the relative separation of the LSB and USB spectral windows in Band 3 is more than $10 \%$ of the band's typical frequency, and because it is possible to obtain sufficient SNR in each sideband of the Band 3 observations; it was decided by the calibrator group to report flux densities separated for both sidebands. The rest of the data consist mostly of observations at Band 6 and measurements taken at different frequencies within Bands 3 and 7. In addition, very sporadic observations at Band 4 were taken for sources J0334-4008, J1733-1304, and J2056-4714.

Using these data we can fit a simple spectral model defined by

$$
\ln \left(\frac{F_{\nu}}{\mathrm{Jy}}\right)=\ln \left(\frac{F_{\nu_{0}}}{\mathrm{Jy}}\right)+\alpha \ln \left(\frac{\nu}{\nu_{0}}\right),
$$

where $\alpha$ is the spectral index and $\nu_{0}$ is a fiducial frequency arbitrarily chosen to be $100 \mathrm{GHz}$. Using model (1), we obtain least squared fitted parameters to all the data available for each calibrator, disregarding its variability. These least-squares best-fit parameters represent time-averaged values, and we denote them $\left\langle F_{100}\right\rangle$ and $\langle\alpha\rangle$, given in columns (7) and (8) of Table 1. Finally, column (9) shows the variability index (vix, Bonato et al. 2018) defined as

$$
v i x=\frac{100}{\langle S\rangle \sqrt{N}} \sqrt{\sum\left(S_{i}-\langle S\rangle\right)^{2}-\sum \sigma_{i}^{2}},
$$

where $\langle S\rangle$ is the time-averaged flux density of the source, calculated at $100 \mathrm{GHz}$ using the fitted law defined by $(1)$, and $\sigma_{i}$ is the observational uncertainty taken from the source catalog. The vix provides a quick quantitative estimation of the percentage of relative time-variability of the source.

Figure 1 shows the data of the source J0635-7516, which will be used as a representative example of the data obtained for the grid calibrators. Similar plots for the rest of the sources in Table 1 can be found as supplementary material (Appendix C). In this work, we adopt the approach followed by previous optical/IR studies of quasar variability which have used the magnitude (or logarithmic) scale as it is better adapted for Gaussian stochastic modeling (e.g., Liodakis et al. 2017, Kushwaha et al. 2016, Edelson et al. 2013, Kelly et al. 2009). In the case of the mm/sub-mm data 
of the grid calibrators, there are two additional reasons: as shown in Figure 1, the lightcurve of Bands 3 and 7 data looks similar but displaced by a constant amount in the log-lin plot, which suggests a relative flux change with a stable spectral index. This type of relative variability is more simply modeled as additive quantities in the logarithmic scale. The second reason is that the main source of flux uncertainty likely arises from multiplicative (calibration-type) errors. Typically, for these bright sources the additive, thermal noise uncertainty does not rise above 1\% (van Kempen et al. 2012).

According to Table 1, most sources (35 from 39) have fluxes projected at $100 \mathrm{GHz}$ below $6.5 \mathrm{Jy}$, with four sources above $9 \mathrm{Jy}$. The spectral indices range between -0.2 and -1.1 , with 32 out of 39 spectral indices between -0.35 and -0.80 . The $100 \mathrm{GHz}$ vix spans between 6.7 and $60.7 \%$. The first, second, and third quantiles of vix of the sources with monitoring times within $30 \%$ of 800 days in the source frame given by Bonato et al. (2018, Table 2) are 13.9, 23.9, and 32.8. These values are comparable with the same quantiles of the vix of those sources in Table 1, with monitoring times compatible with the 800 days criterion, which are 13.5, 19.0, and 37.3, respectively. Since the flux and spectral indices of these sources are variable, the mean spectral index (1) is highly dependent on the time-frequency sampling, which is typically very different between the calibrator survey and the study by Bonato et al. (2018). In any case, the absolute differences in mean spectral index between Table 1 and those calculated for the data presented in Bonato et al. (2018) have a median of 0.04, and are in all cases $<0.24$. Figure 2 shows histograms of $\left\langle F_{100}\right\rangle,\langle\alpha\rangle$, and vix for the grid sources.

Another important characteristic of the quasar variability is the apparent lack of periodic components (e.g. Goyal et al. 2018). A method to assess the presence of (quasi-) periodicity in the data applicable to irregular time sampling is to examine its LombScargle periodogram (VanderPlas 2018). This type of periodogram is analogous to a Fourier transform, and is sensitive to periodic behavior of the auto-covariance function. The red lines in the top and bottom panels of Figure 3 show the $91.46 \mathrm{GHz}$ flux densities of J0635-7516 and its periodogram, respectively. We calculate the periodogram using the lsp task within the $\mathrm{R}$ software ( $\mathrm{R}$ Core Team 2018). It is patent that the only conspicuous peak occurs at zero time-frequency, after which the periodogram power decreases toward higher time-frequencies. This same behavior can be reproduced with stochastic models with autocorrelation, but without any periodicity. For example, the green line in Figure 3 shows a random realizationof an OU-process (see section 3) with decorrelation time $400 \mathrm{~d}$ and variance rate 0.015 , sampled evenly every 7 days. The parameters of the process were selected to nearly match the variability index of the data shown in Table 1.

We stress on a note of caution: one noticeable difference between the periodograms of the green model and the data is that the power of the latter seems to plateau at time-frequencies above $0.01 \mathrm{~d}^{-1}$ at $\approx 1(\mathrm{ln} \mathrm{Jy})^{2}$, whereas the periodogram power of the model decreases toward zero more steadily. This may be in part due to the presence of nearly uncorrelated white noise in the data of the quasar (e.g., instrumental errors), but we find that the main explanation is probably due the inhomogeneous sampling. 

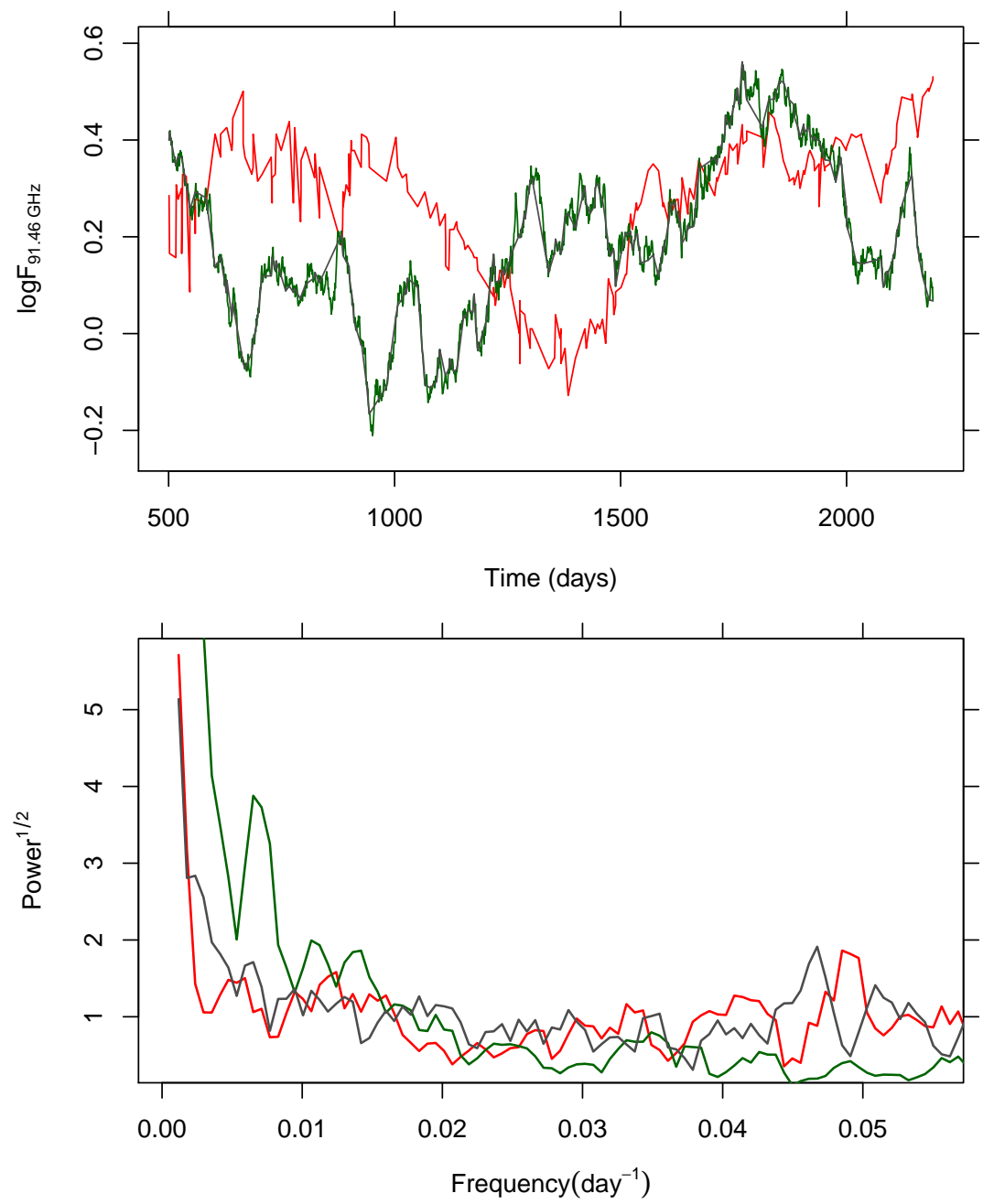

Figure 3. Top panel. Red curve shows the log-flux time-curve of J0635-7516 at 91.46 GHz. Days in the abscissa are taken with respect to the start day of observations (Table 1). Green curve shows a random realization of an OU process with decorrelation time $500 \mathrm{~d}$ and variance rate 0.015 (see section 3), sampled every day. Gray curve shows a random realization of the same OU-process sampled at the same times as the red curve. Bottom panel. Lomb-Scargle periodograms of the data on top panel (same color coding).

This is illustrated by the gray curves in Figure 3, which show another realization of the same OU-process as the green model, but sampled at the times of the red data curve. Similar to the periodogram power of the data, the gray curve decreases much more slowly toward zero compared with the green curve, and both display an excess of power around $0.05 \mathrm{~d}^{-1}$ - which naively may hint to quasi-periodicity — but it is an effect attributable only to the inhomogeneous sampling. 


\section{Methods}

To analyze the time-series data of the calibrator, we follow an additive "classical" decomposition approach (Brockwell \& Davis 2002). According to this notion, we can express the log-flux (or any other adequate function of the flux) at time $t$ and frequency $\nu$ as the sum

$$
\ln F_{\nu}(t)=P_{\nu}(t)+y_{\nu}(t)
$$

where $P_{\nu}(t)$ and $y_{\nu}$ are the 'deterministic' and 'stochastic' models, respectively. The flux density $F_{\nu}$ unit is Jy, unless stated otherwise. $P_{\nu}$ and $y_{\nu}$ are characterized by several parameters, which need to be estimated from the data (e.g., through maximum likelihood estimation).

The frequency dependence of the deterministic models consist of (possibly slightly modified) power laws in frequency. For this study, we assume that the stochastic model is stationary and centered (i.e., zero expectation). Non-stationary features like trends (that is, a long-term increase/decrease of flux) or seasonal (periodic) components can be included in the deterministic modeling.

The stochastic model is based on the OU-process, used to describe the light-curves of quasars by Kelly et al. (2009). Subsequent studies have confirmed this is a reasonably adequate and simple model for the stochastic nature of the light-curves of many quasars (Kozłowski et al. 2010, MacLeod et al. 2010, Ruan et al. 2012, Andrae et al. 2013, Wang \& Shi 2019). It has been found, however, that sometimes this model is too simple to describe the variability on very high cadency data $\left(\sim 10^{3} \mathrm{~s}\right.$, Kasliwal et al. 2015). Extensions to the simple OU-process have been proposed by, for example, Kelly et al. (2011) (finite mixtures), Kelly et al. (2014) (continuous auto-regressive mean-average, CARMA), and Takata et al. (2018) (infinite mixtures).

In its most general form, the stochastic models proposed in this work consist of finite mixtures of OU-processes (Kelly et al. 2011) for the log-flux of the quasar at a fiducial frequency and for its spectral index, plus uncorrelated noise. An important difference between the modeling performed in previous studies and this one is the inclusion of a spectral deterministic model for the source, which allow us to fit together the data at different frequencies within a single model. In contrast, the stochastic analysis of multi-frequency data is done usually (e.g., Goyal et al. 2018) on monochromatic lightcurves. We will denote the process including a mixture of $r$ OU-processes for the fiducial $\log$-flux and $s$ OU-processes for the spectral index as OU-rFsa. In the literature, one of the extensions of a single OU-process is a CARMA process. An OU- $r$ F sa process is equivalent to a CARMA $(r+s, r+s-1$ ) process (Kelly et al. 2014, Granger \& Morris 1976) associated with a characteristic equation with only real solutions.

We can gain insight of the time-series methods applied in this paper analyzing one of its simplest models: the Gaussian autoregressive (AR) model (Scargle 1981). In Appendix A we present a short description of the $\mathrm{AR}(1)$ process, which is an evenly sampled, discrete time, and noiseless version of the stochastic processes we describe 
in the following sections. The main characteristics, advantages, and demerits of the modeling we propose in this work can be generally understood from the AR(1) process.

\subsection{Ornstein-Uhlenbeck mixture models.}

3.1.1. Constant spectral index: OU-1F0a process. We start describing the simplest mixture model: one OU-process characterizing the log-flux at a fiducial frequency, and using (1) for the fluxes at other frequencies. This OU-1F0a model is defined by

$P_{n}=\mu+\alpha \ln \left(\nu_{n} / \nu_{0}\right)$,

$y_{n}=f_{n}+\sigma_{N}\left(\nu_{n}\right) Z_{n}$

$f_{n}=e^{-\delta t_{n} / \tau} f_{n-1}+\sqrt{\frac{\varsigma^{2} \tau}{2}\left(1-e^{-2 \delta t_{n} / \tau}\right)} Z_{n}^{\prime}$.

Equation (4) defines the deterministic model $P_{\nu}$ measured at time $t_{n}(n \geq 1)$. The observed frequency at time $t_{n}$ is $\nu_{n}$. The deterministic model depends on two parameters: the spectral index $\alpha$ and the long-term mean log-flux at the fiducial frequency $\left(\nu_{0}=100 \mathrm{GHz}\right), \mu$. In Equation (5), the stochastic component $y_{n}$ (see (3)) consist of the sum of a Gaussian noise of variance $\sigma_{N}^{2}\left(\nu_{n}\right)$ and a time variable term $f_{n}$. We introduce heteroscedasticity in the model assuming that the noise term $\sigma_{N}(\nu)$ depends on frequency as $\nu^{1 / 2}$. This dependence roughly matches the estimated ratio of the flux density uncertainty at Band 7 and Band 3, which is $\approx 2$ (Warmels et al. 2018, $\S 10$ ). The ratio between the average flux uncertainties at Band 7 and Band 3 given in the source catalog is $2.1 \pm 0.4$, where the error represents the dispersion among the grid sources.

The $f_{n}$ term follows an OU process, defined explicitly in (6), where the time between observations is $\delta t_{n}=t_{n}-t_{n-1}$. Both $\left(Z_{n}, Z_{n}^{\prime}\right)$ are a series of uncorrelated normal standard random variables. The stochastic parameters of the model are three:

$\varsigma^{2}$, the variance rate (time ${ }^{-1}$ units),

$\tau$, the decorrelation time (time units),

$\sigma_{N}^{2}\left(\nu_{0}\right)$, the uncorrelated ("measurement") noise at the fiducial frequency.

Equations (4) to (6) also form a state space representation (see Appendix B) of the stochastic process $\ln F_{\nu}(t)$, in which the "state" role is taken by $f_{n}$. The variance rate is called this way because for $\delta t_{n} \ll \tau$, the variance of $\ln F_{0}\left(t_{n}\right)$ assuming $\ln F_{0}\left(t_{n-1}\right)$ is given by $\varsigma^{2} \delta t_{n}$. Note that it is not rare to have simultaneous measurements (taken during the same day, in our case) of the source at different frequencies. These observations can be included as part of the time series in any order. They cannot give us information about the time variability, but they are useful to estimate the uncorrelated noise level $\sigma_{N}$

3.1.2. OU-rFs a plus noise process. This is a more generic model than the one presented in the previous section. It consists of a mixture of OU-processes for the flux and for the 
spectral index. They are defined by

$$
\begin{aligned}
& P_{n}=\mu+\alpha \ln \left(\nu_{n} / \nu_{0}\right) \\
& y_{n}=\sum_{i=1}^{r} f_{n}^{i}+\sum_{j=1}^{s} \beta_{n}^{j} \ln \left(\nu_{n} / \nu_{0}\right)+\sigma_{N}\left(\nu_{n}\right) Z_{n}, \\
& \left(\begin{array}{c}
\mathbf{f}_{n} \\
\boldsymbol{\beta}_{n}
\end{array}\right)=e^{-\delta t_{n} \boldsymbol{\tau}^{-1}}\left(\begin{array}{c}
\mathbf{f}_{n-1} \\
\boldsymbol{\beta}_{n-1}
\end{array}\right)+\eta_{n}, \\
& \boldsymbol{\tau}=\operatorname{diag}\left(\tau_{1}, \ldots, \tau_{r}, T_{1}, \ldots, T_{s}\right), \\
& \boldsymbol{\Sigma}=\operatorname{diag}\left(\varsigma_{1} \ldots, \varsigma_{r}, \zeta_{1}, \ldots, \zeta_{s}\right), \\
& \eta_{n} \sim \operatorname{GWN}\left(\mathbf{0}, \int_{t_{n-1}}^{t_{n}} e^{\left(t_{n}-s\right) \boldsymbol{\tau}^{-1}} \mathbf{\Sigma} \Gamma \boldsymbol{\Sigma} e^{\left(t_{n}-s\right) \boldsymbol{\tau}^{-1}} d s\right) .
\end{aligned}
$$

In (7), the deterministic model is the same as in the previous section, but $\alpha$ in this context represent the long-term mean spectral index. In (8), $f^{i}$ and $\beta^{j}$ are centered OU-processes and the components of the vectors $\mathbf{f}$ and $\boldsymbol{\beta}$, respectively. The definition of the OU-process mixture is given by Equations (9) to (12). In Equations (10) and (11) $\operatorname{diag}(\ldots)$ represents a diagonal matrix, with the listed values as the diagonal elements. Finally, in (12) $\Gamma$ is a symmetric $(r+s) \times(r+s)$ matrix representing cross-correlations between the terms, with $\left|\Gamma_{k, l}\right|<\Gamma_{k, k}=1$ for all $k, l=1, \ldots,(r+s), k \neq l$. Decorrelation times and variance rates are given by $\tau_{i}\left(T_{j}\right)$ and $\varsigma_{i}\left(\zeta_{j}\right)$ for each $f^{i}\left(\beta^{j}\right)$, respectively. In (12), GWN stands for Gaussian white noise, meaning that $\eta_{n}$ forms a series of uncorrelated - in time - multivariate normal variables. We emphasize that the model includes normal variables in two instances: as a "noise" term in (8), and as part of the OU-process in (12). Similarly as in section 3.1.1, Equations (8) to (12) define a state space representation of the measurements with a state vector $\left(f^{1}, \ldots, f^{r}, \beta^{1}, \ldots, \beta^{s}\right)^{\mathrm{T}}$.

In practice, for this work we use $r \leq 2$ and $s \leq 1$ models. Note that the matrix $\Gamma$ defines a set of additional $(r+s)(r+s-1) / 2$ parameters which need to be adjusted or assumed. While choosing $\Gamma$ equal to the identity matrix - that is, no cross-correlations - might look like a natural choice, we find that it has the undesirable consequence of making the model too dependent on the fiducial frequency. Indeed, at the fiducial frequency the model would be characterized by only $f$ terms, and no $\beta$ terms, effectively reducing the order of the process. Since the fiducial frequency $(100 \mathrm{GHz})$ is arbitrarily chosen, this dependence is unjustified. Including cross-correlation terms allows us to moderate this artificial and unbalanced role given to the fiducial frequency. Ultimately, the reason behind this complication lies in the difficulty of describing a varying spectral index independently of flux variations, since these are correlated. An alternative to include cross-correlations may be to leave the fiducial frequency $\nu_{0}$ as a free parameter, possibly even time-variable. Exploring all these alternatives, however, is beyond the scope of this work.

For a general OU-1F1a process (dropping the $i=j=1$ indices), the crosscorrelation between $f_{n}$ and $\beta_{n}$ is defined by a single parameter $\rho$, with $\Gamma_{1,2}=\Gamma_{2,1}=\rho$. 
Hence, $\eta_{n}$ is a centered bi-normal variable with covariance matrix

$$
\left[\begin{array}{cc}
\frac{\varsigma^{2} \tau}{2}\left(1-e^{-2 \delta t_{n} / \tau}\right) & \rho \frac{\varsigma \zeta \tau T}{\tau+T}\left(1-e^{-\left(\frac{\delta t_{n}}{\tau}+\frac{\delta t_{n}}{T}\right)}\right) \\
\rho \frac{\varsigma \zeta \tau T}{\tau+T}\left(1-e^{-\left(\frac{\delta t_{n}}{\tau}+\frac{\delta t_{n}}{T}\right)}\right) & \frac{\zeta T^{2}}{2}\left(1-e^{-2 \delta t_{n} / T}\right)
\end{array}\right] .
$$

3.1.3. Additional features. According to the prevalent physical model of blazars, their emission is mainly synchrotron radiation arising from a relativistic jet, directed nearly in our line of sight, and powered by accretion onto a supermassive black-hole (Blandford \& Königl 1979, Begelman et al. 1984). Shocks within the jet may increase the luminosity of the jet, and they may be the root cause of some of the observed variability (Ulrich et al. 1997). Based more or less on this physical picture, we study two more additions to the model which help to fit better the quasars light-curves (e.g., Trippe et al. 2011). These are:

Spectral curvature.

Synchrotron cooling may cause a decrease in the spectral index of the emission starting at high frequencies, an effect also known as "ageing" of the spectrum (Kellermann \& Verschuur 1988). We can generalize the spectral model for $P_{\nu}$ in the following way (e.g., Xue et al. 2016)

$$
P_{\nu}=\mu+\left(\alpha+\kappa \ln \left(\nu / \nu_{0}\right)\right) \ln \left(\nu / \nu_{0}\right),
$$

which amounts to a spectral index varying with frequency. The sign of $\kappa$ being negative or positive defines what we call concave and convex spectral models, respectively. The local spectral index at frequency $\nu$ is

$$
\frac{d \ln \left(F_{\nu}\right)}{d \ln \left(\nu / \nu_{0}\right)}=\alpha+2 \kappa \ln \left(\nu / \nu_{0}\right)+\sum_{j=1}^{s} \beta^{j}\left(t_{n}\right)
$$

Frequency depending lag ( $\nu$-lag).

A common interpretation of the $\mathrm{mm} / \mathrm{sub}-\mathrm{mm}$ blazar variability is provided by the shock-in-jet model (Sokolov et al. 2004, Joshi \& Böttcher 2011, Hada et al. 2011). According to it, flares of various intensities are generated by the development of shock waves traveling downstream through the jet body. This type of model produces naturally time-variable emission features with frequency depending light-curves (e.g., Fromm et al. 2015). Light-curves with comparable frequencies display qualitatively similar morphology, but with a time displacement or delay dependent on frequency. Therefore, we add another potential feature to the blazar light-curves by mixing frequency and temporal coordinates, that is,

$$
y_{n}(t, \nu)=y_{n}(t+\Delta t(\nu))
$$

where $\Delta t(\nu)$ is a frequency dependent time lag, chosen to be $\Delta t(\nu)=\epsilon \ln \left(\nu / \nu_{0}\right)$. A positive $\epsilon$ is associated with corresponding variations at frequencies higher than $\nu_{0}$ occurring at later times than those observed at $\nu_{0}$, and vice-versa.

In the shock-in-jet model, the $\mathrm{mm} / \mathrm{sub}-\mathrm{mm}$ emission arises mainly from synchrotron. Due to the synchrotron opacity increasing upstream in the jet and 
decreasing with frequency, we expect higher-frequency emission tracing an earlier development of the shock wave. That is, the most common physical interpretation predicts only negative $\epsilon$, as it is commonly observed (Fuhrmann et al. 2014). We must note also that there is the possibility of degeneracy between the $\nu$-lag and a variable spectral index, specially affecting observations at only two frequencies. For example, a short flare led by the high-frequency flux can be equally well described by a sudden increase and later decrease of the spectral index. Alternatively, a decrease and later increase in the spectral index may be interpreted as a positive lag. We test the ability of the model to resolve this degeneracy with data at only two bands in Section 3.4. For the current modeling, we leave the sign of $\epsilon$ free and evaluate its reliability later.

\subsection{Determination of the best-fit stochastic model.}

Estimation of the best-fit model parameters and their uncertainties is done through likelihood maximization. To calculate the likelihood function we use the state space representation of each process together with the Kalman recursions, as described in Appendix B. The Kalman recursions (Hamilton 1994) are a group of recursive equations which allow us to obtain best estimators (in the least squares sense) of forecasts and interpolations of stochastic time series. For Gaussian time series, Kalman recursions provide the conditional expectations of forecasts and interpolations given the observed data.

Briefly, the procedure to obtain the maximum likelihood estimators (MLE) has the following steps

1. For each set of parameters, subtract from the log-fluxes the deterministic model.

2. Calculate the log-likelihood as a function of the stochastic parameters and the Kalman predictions (B.11) (Appendix B).

3. Re-iterate 1-2 in order to maximize the log-likelihood with respect to the free parameters. We use the Minuit (James \& Roos 1975) package implemented within PDL $\S$ to obtain the MLE and their uncertainties.

3.2.1. Quality of the best-fit model. Testing the hypothesis that the data originated from a certain stochastic model entails evaluating the fit according to the following criteria (e.g., Koen 2005):

(i) The Akaike information criterion (AIC, Feigelson \& Babu 2012). Defined as $2 k-2 \ln \mathcal{L}_{\max }$, where $k$ is the number of free parameters of the model and $\mathcal{L}_{\max }$ is the maximum attained likelihood. The AIC allows us to compare between models and make a first evaluation about the merit of including more free parameters in the model. Better-fit models are associated with a lower AIC.

$\S$ http://pdl.perl.org/ 
(ii) Normalized residual plot. We define the normalized residuals of the observed stochastic components $y_{n}$ with respect to the best-fit predictions $y_{n \mid n-1}$ by

$$
\chi_{n}=\frac{y_{n}-y_{n \mid n-1}}{\sqrt{r_{n}}},
$$

where the notations follows from (B.10) (Appendix B), that is, $y_{n \mid n-1}$ is the best forecast of the $\mathrm{n}$-th measurement and $r_{n}$ is the uncertainty of this forecast. We inspect the plot of residuals vs. time coloring them by band in order to identify any systematic effect depending on frequency or time, for example, a change in spectral index.

(iii) According to the hypotheses, $\chi_{n}$ in (17) should resemble a standard GWN series for large $n$ (Kelly et al. 2011). We test the normality of the residuals running the Anderson-Darling test (Feigelson \& Babu 2012) using the ad.test task from package goftest (Faraway et al. 2017). We also examine the histogram of $\chi_{n}$ and compare it with a standardized Gaussian probability density.

(iv) We test the hypotheses that $\chi_{n}$ forms an uncorrelated white noise series by calculating its autocorrelation function (ACF) using the task acf from R. For a white noise sequence, the only significant peak of the ACF should be located at zero.

The AIC is more useful as a relative criterion to compare between different models, while the other three evaluate the goodness-of-fit. These goodness of fit criteria test how well the stochastic and deterministic models can describe the calibrators light-curves, leaving final residuals consistent with Gaussian white noise. Additional features may provide a slightly better fit to the data (model more adequate) but at the expense of introducing excessive sophistication and degeneracy between the parameters (less parsimony). Some heuristic assessment of the merits of the optimized models is necessary to finally decide which is the best model for each source. From the best-fit model, using the Kalman recursions (Appendix B) we derive predictions and interpolations for the process at arbitrary time, together with its uncertainties.

\subsection{Uncertainty of the predictions}

There are three types of uncertainty associated with the predictions or forecasts made by the model. The first two are determined by $\sigma_{N}(\nu)$ (what we call measurement noise), and the stochastic variability, determined by the variance rates and decorrelation times through the Kalman recursions (Equation (B.10) in Appendix B). The modeling assumes these two types of uncertainty are uncorrelated, and its sum in quadrature is denoted by $r_{n}$ (Appendix B). This would be the only source of uncertainty for a process with perfectly determined parameters. The third type of uncertainty is derived from the uncertainty associated with the best-fit parameters.

The actual flux prediction is not given by $y_{n \mid n-1}$, but by $P_{\nu_{n}}+y_{n \mid n+1}$ (3). In both the deterministic model $P_{\nu}$ and in the stochastic model, best-fit parameters have been 
estimated from the data itself. Therefore, there is an error level associated with the ambiguity about which model best fit the data. We refer hereafter to this forecast (or interpolation) error level as the "parametric" uncertainty. Uncertainties of the MLE parameters can be estimated using their covariance matrix calculated by Minuit using the second derivative matrix of the likelihood function at maximum. When the fit performs poorly and the likelihood is very non-Gaussian - which happens, for example, when the decorrelation times are long — the covariance matrix is still useful as an order of magnitude estimator.

To estimate the parametric uncertainty of the forecast, we consider the estimators as random variables around the MLE solution with the covariance matrix describing its uncertainty. This random variable is taken as independent of any single measurement (particularly, the last one), a hypothesis valid for large number of samples. As a simplified example, Appendix A discusses the parametric uncertainty associated with an unknown long-term mean $\mu$ for an AR(1) process. An estimation of this error term is given in (A.10). These formulae quantify the intuitive idea that the most relevant information in order to make a forecast is the last (that is, the most recent) measurement. However, if this last sampling was taken too long ago (several decorrelation times), it is no longer useful as a predictor, and the best forecast becomes the long-term mean with an error comparable to the source's observed variability.

In order to obtain an explicit parametric uncertainty estimation, we construct our forecast using the Kalman filter (Appendix B) using only the last measurement. We also make the assumption that there is no covariance between the $f$ and $\beta$ terms. The forecast at time $t$ and frequency $\nu$, based on a single flux $F_{1}$ measured at time $t=0$ and at frequency $\nu_{1}$, is given by

$$
P_{\nu}+\left(\ln F_{1}-P_{\nu_{1}}\right) \frac{H e^{-t \tau^{-1}} C H_{1}^{\mathrm{T}}}{\sigma_{N}^{2}(\nu)+H_{1} C H_{1}^{\mathrm{T}}},
$$

where $C=\frac{1}{2} \operatorname{diag}\left(\varsigma_{1} \tau_{1}^{2}, \ldots, \varsigma_{r}^{2} \tau_{r}, \zeta_{1}^{2} T_{1}, \ldots, \zeta_{s}^{2} T_{s}\right)$ and $H$ is given by (B.4) in Appendix B. Let the gradient of (18) respect to the parameters be $\boldsymbol{J}$. Then, the uncertainty of the forecast of $\ln F_{\nu}(t)$ is

$$
\boldsymbol{J} \Sigma_{\mathrm{MLE}} \boldsymbol{J}^{\mathrm{T}}
$$

where $\Sigma_{\text {MLE }}$ is the covariance matrix at the maximum likelihood (e.g., Bevington \& Robinson 2003). Finally, for simplicity, we calculate the total uncertainty on the forecast as the sum in quadrature of the parametric and stochastic uncertainties.

\subsection{Performance in simulated data}

We test the ability of the MLE procedure to recover the parameters of simulated, irregularly sampled data, under the hypotheses described in previous sections. In order to do this, we generate $100 \mathrm{OU}-1 \mathrm{~F} 1 \mathrm{a}$ simulated light-curves at 91.5, 103.5, and 343.5 $\mathrm{GHz}$ with parameters $\mu=0, \alpha=-0.7, \epsilon=-2(\mathrm{~d}), \kappa=-0.1, \sigma_{N}=0.04 \sqrt{\nu / \nu_{0}}, \tau=360$ $\mathrm{d}, T=50 \mathrm{~d}, \varsigma=0.05 \mathrm{~d}^{-1 / 2}, \zeta=0.01 \mathrm{~d}^{-1 / 2}$, and monitoring time $1080 \mathrm{~d}$. The curves 
have 400 measurements each with a $30 \%$ of the fluxes in Band 7, and the rest in Band 3 , which is comparable to the frequency breakdown of the real data. We intentionally take the monitoring time covering only three decorrelation times for the $f$ component in order to explore the effects of a poorly constrained $\tau$ (Kozłowski 2017b). We analyze the consequences of this particular limitation in more detail in Section 5 and Appendix A. The simulated parameters produce light-curves with a similar appearance compared with those of the grid calibrators. One example of these curves (dubbed J2600+0010) is shown in the top panel of Figure 4, and a $\mathrm{R}$ script is provided in the supplementary material to produce this one and the rest of the simulated light-curves.

Figure 5 shows box-plots of the differences between MLE recovered values and simulated ones for the 100 synthetic light-curves. We find that the parameters describing the spectral shape of the source (like the spectral index and curvature parameter) are in general well recovered by MLE, with little bias. As expected, $\tau$ (not shown in Figure 5) is not recovered accurately. The median value of the MLE $\tau$ is $170 \mathrm{~d}$, not close the value of the simulation. For $T$, the MLE median is $45 \mathrm{~d}$, but with a very large dispersion. Large uncertainties in the decorrelation times also imply large uncertainties in the longterm mean estimations. Indeed, as shown in Figure 5, the MLE of $\mu$ has a relatively large dispersion respect to the simulated value, although not obviously biased. The rest of the parameters seems to be reasonably well recovered, with the MLE uncertainty providing a sensible scale for its dispersion.

The simulations also allow us to estimate the behavior of the model in an idealized scenario. Based on these results, we do not expect the fitting to recover the spectral index and its curvature parameter with an uncertainty better than 0.05 , and the $\nu$-lag parameter $\epsilon$ with an uncertainty better than $0.5 \mathrm{~d}$, despite the MLE uncertainty of these sometimes suggesting even smaller error bars. Considering also that the time resolution of the light-curves is $1 \mathrm{~d}$, a minimum uncertainty of $0.5 \mathrm{~d}$ in the $\epsilon$ parameter seems sensible.

Figure 4 also shows the simulated and recovered $\mu+f_{n}$ and $\alpha+\beta_{n}$ for $\mathrm{J} 2600+0010$ in the middle and bottom panel, respectively; the latter showing the variable spectral index of the simulated quasar. While $\mu+f_{n}$ is obtained very well by MLE, the spectral index seems less well recovered. The MLE spectral index curve follows only qualitatively the simulated variable spectral index, with typical differences within 0.05 . Note, however, that differences of this same order of magnitude are seen in the middle panel, but they are less noticeable due to the larger dynamic range.

Part of the difficulties to recover the spectral index history may also be related to the need of observing at more than one frequency to get basic spectral information. Band 3 observations are more frequent than those at band Band 7. The monitoring is somewhat inhomogeneous due to observational circumstances, but we can define a mean cadency given by the average separation in days between consecutive observations. Among the grid sources, the median separation between consecutive Band 7 observations is 17 days, while for Band 3 observations is 12 days. Henceforth, we do not expect to recover spectral index features on timescales shorter than the mean cadency of the Band 

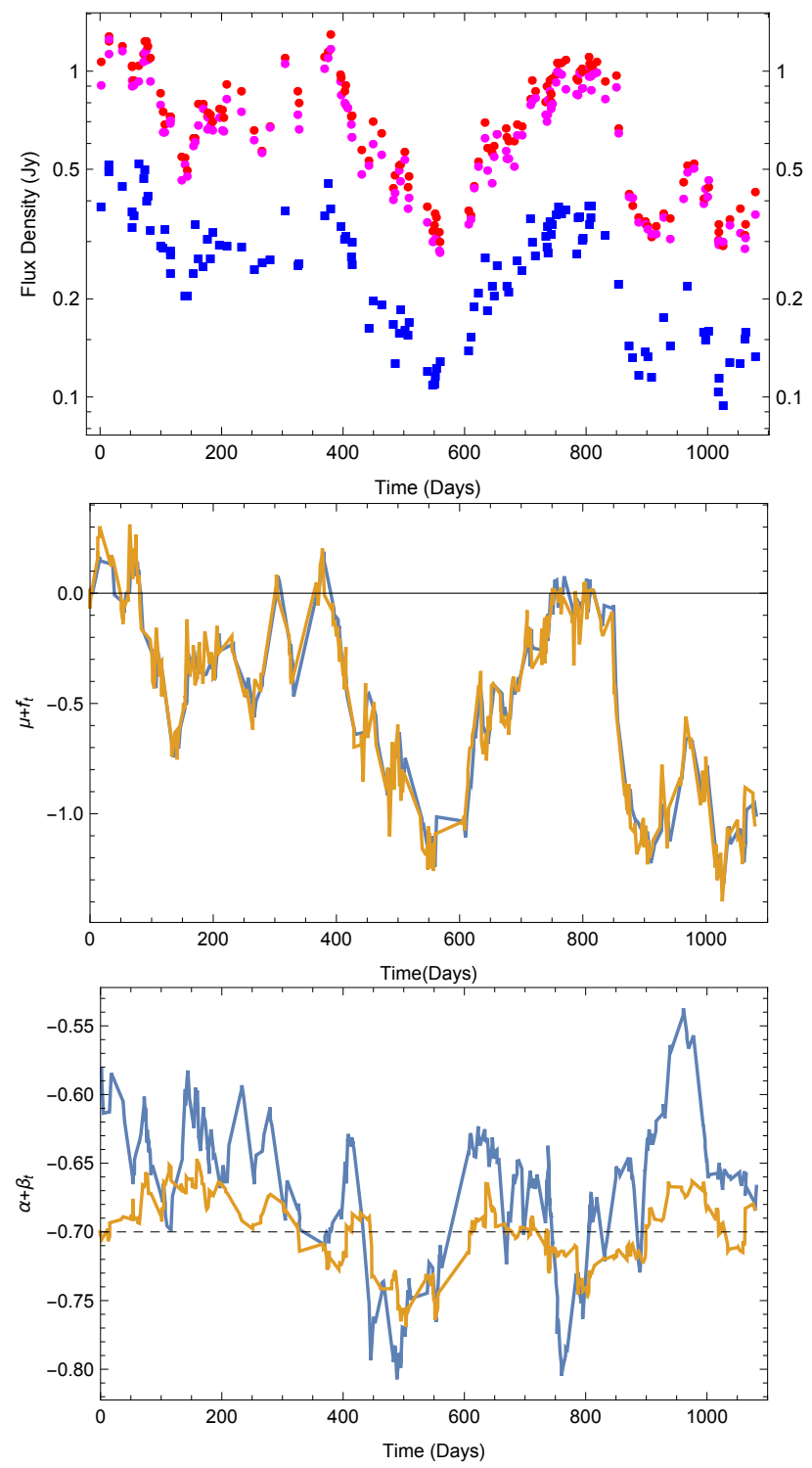

Figure 4. Top panel. One example (J2600+0010) of the 100 simulated OU-1F1a light-curves at 91.5 and $103.5 \mathrm{GHz}$ (in red and magenta circles, respectively), and $343.5 \mathrm{GHz}$ (blue squares). Middle panel. Blue and orange lines show the simulated and MLE $\mu+f_{n}$ curves, respectively. Bottom panel. Same as the previous panel but showing the $\alpha+\beta_{n}$ curves.

7 monitoring.

\section{Results}

\subsection{Best fit models.}

As a first step we fit the OU-1F0a model to the grid calibrators. Table 2 lists the best fit parameters for each calibrator. Columns (2) to (6) give the MLE $\mu, \alpha, \sigma_{N, 0}$ $\left(\right.$ at $\left.\nu_{0}\right), \tau$, and $\varsigma$ (see (4) and (6)), respectively. We find that the heteroscedasticity 


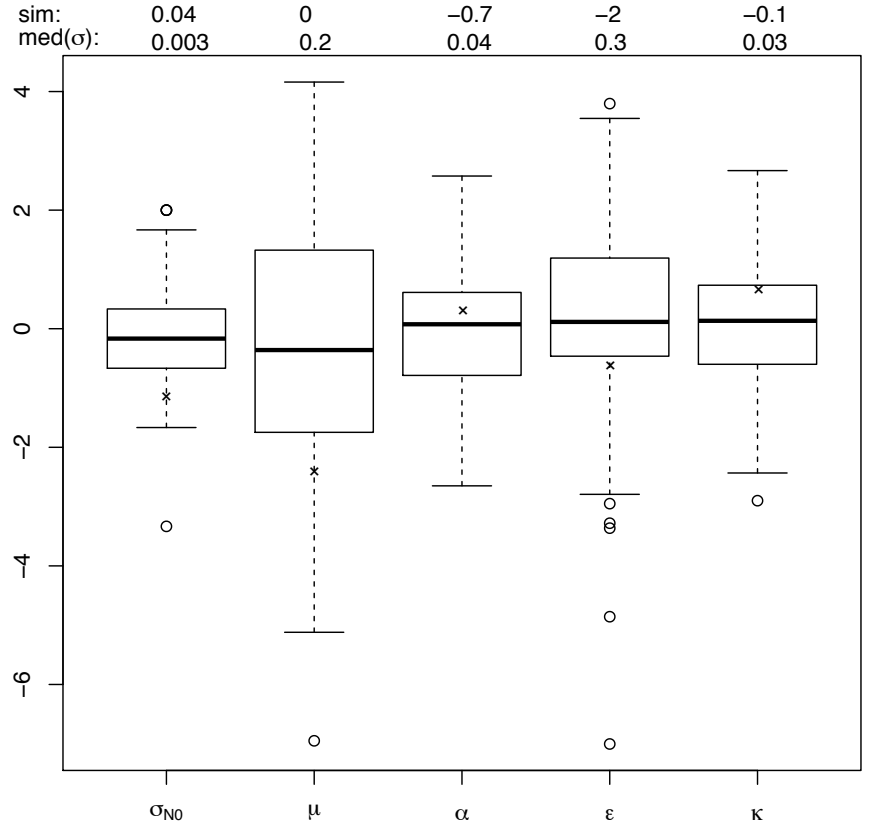

Figure 5. Box-plot (Feigelson \& Babu 2012, Section 5.9) of the scaled differences between the given simulated parameters $\sigma_{N, 0}, \mu, \alpha, \epsilon$, and $\kappa$ (see Section 3.1 .2 and 3.1.3) and their MLE best fit, for the 100 synthetic light-curves. The differences have been scaled by the median MLE uncertainty of each parameter. The values adopted for the simulations and the median MLE uncertainties are given on top of the plot. The box-plot shows the median (thick black line) and the interquartile range as boxes encompassing half (50) of the points. The error bars encompass the data within 1.5 times the inter-quartile distance from the box limits. Points outside these limits are marked individually with circles. The crosses mark the recovered MLE parameters for the simulated light-curve J2600+0010, shown in Figure 4.

prescription of $\sigma_{N}(\nu)$ depending as $\nu^{1 / 2}$ is reasonable, providing in all cases a lower AIC compared either with a single $\sigma_{N}$ (homoscedasticity) or with a heteroscedasticity prescription $\propto \nu$. Because data other than those at Band 3 and 7 are very limited, we do not test extensively other dependences of $\sigma_{N}$ with frequency.

The (1-sigma) parabolic uncertainties in Table 2 have been calculated from the likelihood function around the minimum. Columns (7) and (8) show the AIC and the p-value of the Anderson-Darling test of normality of the residuals ( $\mathrm{p}_{\mathrm{A}-\mathrm{D}}$ ), respectively. Column (9) shows the ratio between the total time span of the monitoring $\Delta T$ versus $\tau$. This quantity is crucial to determine the confidence on the best-fit $\tau$ values (Kozłowski 2017b). Values of $\Delta T / \tau \geq 10$ indicate reliable MLE $\tau$, while for $\Delta T / \tau<5$ the estimation is not reliable at all. Altogether, the formal uncertainties of $\tau$ and $\mu$ are higher for lower values of $\Delta T / \tau$. Also, in this case, the log-likelihood function around the maximum is not symmetric and therefore the parabolic uncertainties only give a rough idea of the parameter error, which may be significantly skewed.

Despite the very simple hypotheses of the OU-1F0a model, it performs well as first approximation in optical data (Kelly et al. 2009) and in the mm/sub-mm data of the grid calibrators. As a representative example, Figure 6 shows the results of the 
OU-1F0a fitting to J0635-7516. The middle and bottom panels of Figure 6 show the time-series of normalized residuals and its histogram, respectively. The histogram and the $\mathrm{p}_{\mathrm{A}-\mathrm{D}}=0.28$ indicates that the aggregated residuals are consistent with the normal hypothesis. In fact, for 27 of the sources the Anderson-Darling test cannot reject the hypothesis of normality at the 0.05 confidence level. Similar plots as Figure 6 for the 39 grid calibrators are given in the supplementary material.

The simple OU-1F0a model is also useful to identify possible outliers in the data. For example, data points located at more than $5 \sigma$ from the best prediction may be considered suspicious, specially if they do not seem to be associated with a more consistent and relatively longer "flaring" type of activity. In any case, censoring data of these varying sources should be performed conservatively. In the case of J0635-7516, we removed 8 data points, corresponding to a $1.06 \%$ of the total amount of data: those measurements taken in 2013-12-29, and the data taken at the non-standard frequency 104.2 GHz at the end of January 2015. The remotion of these points does improve the general quality of the light-curve fittings.

Despite the apparent adequacy of the OU-1F0a model, a more careful analysis of the residuals reveals its shortcomings. For example, the second panel of Figure 6 shows that constant spectral index hypothesis is not the most adequate: there are systematic patterns respect to frequency in the range between 900-1300 d, and specially after day 1800. These systematics are somewhat confused when aggregating all the residuals as in the third panel. Furthermore, the ACF of the $\chi_{n}$ displayed in Figure 7 shows that the residuals separated by Band have significant cross-correlation not consistent with white noise.

Table 3 shows a comparison of different models applied to J0635-7516. The first column shows the OU-mixture model. Curvature and $\nu$-lag refers to the additional features described in Section 3.1.3. Because the AIC is useful as comparison criteria between models, column (4) shows the difference between the AIC attained by the specified model and the OU-1F0a. Columns (5) and (6) shows the $\mathrm{p}_{\mathrm{A}-\mathrm{D}}$ and the p-value of the Ljung-Box test $\left(\mathrm{p}_{\mathrm{L}-\mathrm{B}}\right)$. The null hypotheses of the Ljung-Box test assumes that the residuals arise from GWN.

We see that the addition of stochastic time-variability to the spectral index (a $\beta$ term) accounts for a significant diminishing in the AIC $(\sim 420)$. The second sophistication in importance, judged from the AIC variation, is the addition of curvature — specifically, concavity — which reduces the AIC by $\sim 90$. As shown in Table 3, the inclusion of $\nu$-lag in the model does not seem to improve the AIC, but it does increase $\mathrm{p}_{\mathrm{A}-\mathrm{D}}$ and $\mathrm{p}_{\mathrm{L}-\mathrm{B}}$, indicating that the residuals seem to be better described by GWN. Based on the criteria defined in section 3.2.1, we conclude that the model which is simultaneously simpler and more adequate for J0635-7516 is the OU-1F1a model with lag and additional concavity. Figure 7 shows in the second panel the ACF of the total residuals and those segregated by band of this model applied to J0635-7516, which are much smaller and similar to a GWN compared with those of the OU-1F0a model. Figure 8 show the best-fit flux and spectral index predictions of the model in the 

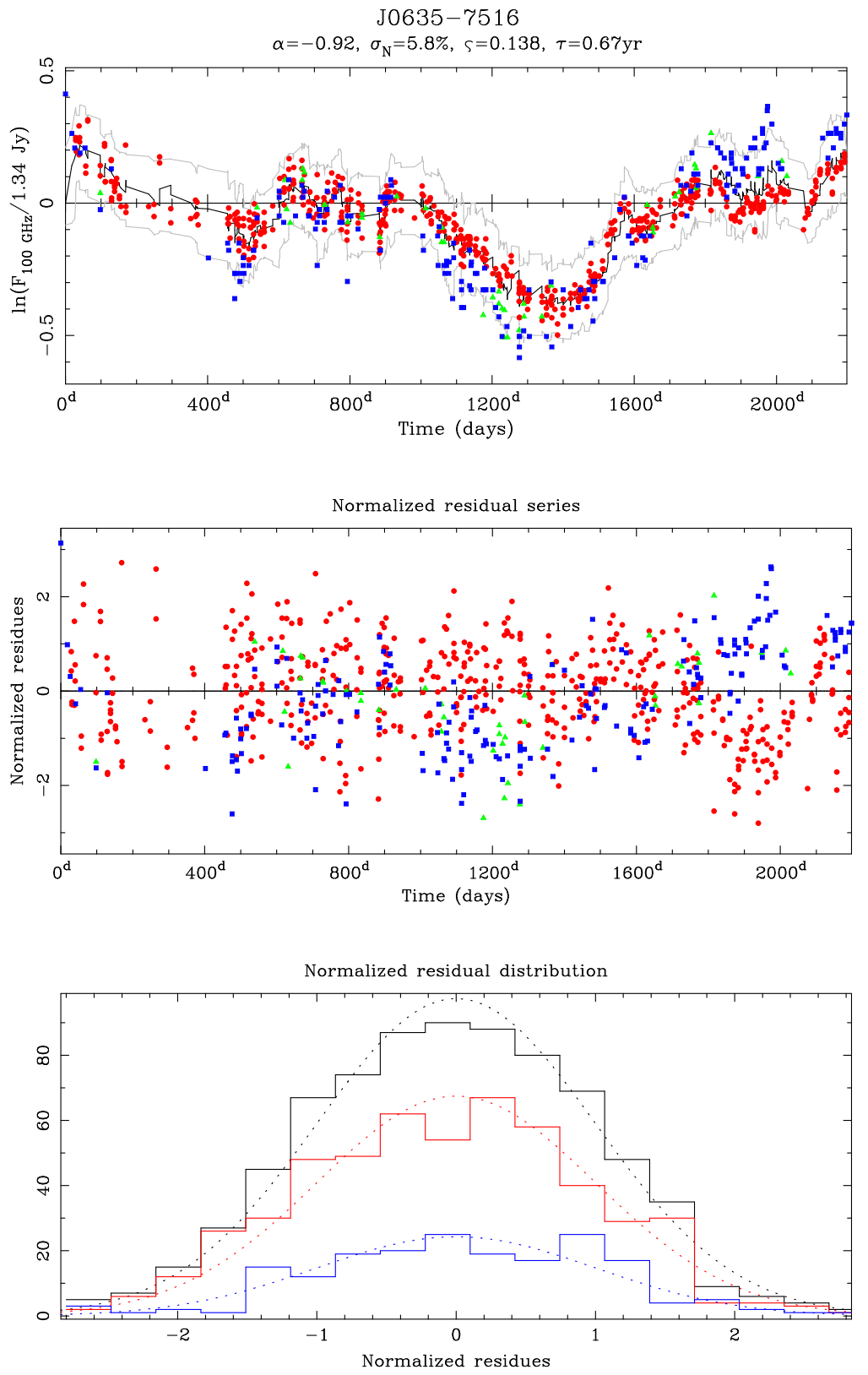

Figure 6. Best-fit OU-1F0a model applied to J0635-7516. Top panel. Centered $\log$-flux curve at $100 \mathrm{GHz}\left(\ln \left(F_{100}\right)-\mu\right)$. Red points and blue squares indicate Band 3 and 7 data, respectively. Green triangles represent data between 116-275 GHz (mostly Band 6, with very sporadic band 4 data). Black line shows the predictions based on previous data $\left(y_{n \mid n-1}\right.$, see (B.10) in Appendix B for notation). Grey lines encloses the 95\% confidence interval around the predictions. Middle panel. Normalized residuals $\chi_{n}$ vs. time (17). Bottom panel. Histograms of $\chi_{n}$. Black is all data, red and blue represent the Band 3 and Band 7 residuals, respectively. Dashed line shows scaled standard Gaussian probability densities. 
Stochastic variability of ALMA calibrators

Table 2. Best-fit OU-1F0a model parameter and results.

\begin{tabular}{|c|c|c|c|c|c|c|c|c|}
\hline Source & $\begin{array}{r}\mu \\
(\ln \mathrm{Jy})^{\mathrm{a}}\end{array}$ & $\alpha$ & $\sigma_{N, 0}$ & $\begin{array}{l}\tau \\
(\mathrm{yr})\end{array}$ & $\varsigma^{\left(\sqrt{\mathrm{yr}}^{-1}\right)}$ & AIC & $\mathrm{p}_{\mathrm{A}-\mathrm{D}}$ & $\frac{\Delta T}{\tau}$ \\
\hline J0006-0623 & $1.161 \pm 0.08$ & $-0.565 \pm 0.006$ & $0.046 \pm 0.002$ & $0.96 \pm 0.3$ & $0.202 \pm 0.02$ & -1017.4 & $5 \mathrm{E}-01$ & 6 \\
\hline $\mathrm{J} 0237+2848$ & $0.758 \pm 0.05$ & $-0.614 \pm 0.005$ & $0.044 \pm 0.002$ & $49 \pm 0.2$ & $260 \pm 0.03$ & -942.0 & $1 \mathrm{E}-01$ & 12 \\
\hline J0238+1636 & $0.34 \pm 0.1$ & $-0.478 \pm 0.005$ & $0.043 \pm 0.002$ & $58 \pm 0.2$ & $.509 \pm 0.04$ & -1047.3 & $4 \mathrm{E}-03$ & 10 \\
\hline J0319+4130 & $2.777 \pm 0.09$ & $-0.711 \pm 0.006$ & $0.043 \pm 0.002$ & $37 \pm$ & $158 \pm 0.02$ & -780.3 & 8.9E-04 & 4 \\
\hline J0334-4008 & $-0.09 \pm 0.8$ & $-0.618 \pm 0.005$ & $0.043 \pm 0.001$ & $8 \pm 10$ & $270 \pm 0.02$ & -1335.7 & $2.1 \mathrm{E}-03$ & 0 \\
\hline J0423-0120 & $1.12 \pm 0.3$ & $-0.588 \pm 0.007$ & $0.071 \pm 0.002$ & \pm 2 & $251 \pm 0.02$ & -1070.6 & $4.1 \mathrm{E}-01$ & 1 \\
\hline J0510+1800 & $0.87 \pm 0.1$ & $-0.411 \pm 0.006$ & $0.053 \pm 0.002$ & $0.65 \pm 0.2$ & $.408 \pm 0.03$ & -943.4 & $1.0 \mathrm{E}+00$ & 9 \\
\hline J0519-4546 & $0.318 \pm 0.08$ & $-0.381 \pm 0.006$ & $0.052 \pm 0.002$ & $0.89=$ & $0.206 \pm 0.02$ & -1184.6 & $4.2 \mathrm{E}-02$ & 6 \\
\hline J0522-3627 & $1.719 \pm 0.05$ & $-0.239 \pm 0.005$ & $0.048 \pm 0.002$ & $0.259 \pm 0.0$ & $458 \pm 0.03$ & -1044.7 & $E-01$ & 23 \\
\hline J0538-4405 & $0.86 \pm 0.2$ & $-0.605 \pm 0.005$ & $0.052 \pm 0.002$ & $5 \pm 1$ & $213 \pm 0.02$ & -1360.3 & $\mathrm{E}-03$ & 2 \\
\hline J0635-7516 & $0.288 \pm 0.04$ & $-0.918 \pm 0.005$ & $0.057 \pm 0.002$ & $0.68 \pm 0.2$ & $0.138 \pm 0.01$ & -1813.2 & 3.0E-01 & 9 \\
\hline J0750+1231 & $0.314 \pm 0.1$ & $-0.657 \pm 0.005$ & $0.039 \pm 0.002$ & $.38=$ & $0.195 \pm 0.02$ & -1055.7 & $1.3 \mathrm{E}-02$ & 4 \\
\hline J0854+2006 & $1.524 \pm 0.08$ & $-0.424 \pm 0.007$ & $0.062 \pm 0.002$ & .1 & $585 \pm 0.04$ & -754.3 & -01 & 17 \\
\hline J0904-5735 & $0.089 \pm 0.1$ & $-0.407 \pm 0$ & $0.049 \pm$ & & $65 \pm 0.1$ & -163.7 & -01 & 20 \\
\hline J1037-2934 & $0.21 \pm 0.1$ & $-0.524 \pm 0.006$ & $0.053 \pm 0.002$ & 0.4 & $0.349 \pm 0.03$ & -1057.1 & 01 & 7 \\
\hline $\mathrm{J} 1058+0133$ & $1.19 \pm 0.2$ & $-0.472 \pm 0.005$ & $0.046 \pm 0.002$ & $2.2 \pm 1$ & $0.248 \pm 0.03$ & -953.7 & 01 & 3 \\
\hline J1107-4449 & $0.163 \pm 0.04$ & $-0.748 \pm 0.006$ & $0.054 \pm$ & $0.53 \pm 0.2$ & $0.170 \pm$ & -1042.1 & 1 & 11 \\
\hline J1127-1857 & $0.09 \pm 0.2$ & -0.5 & 0.0 & & 05 & -283.3 & & 3 \\
\hline $\mathrm{J} 1146+3958$ & 0.1 & -0 & & & 0.361 & -3 & & 6 \\
\hline $\mathrm{J} 1229+0203$ & $2.165 \pm 0.08$ & $-0.775 \pm 0.007$ & 0. & 0 & $0.262 \pm$ & -942.1 & 01 & 8 \\
\hline $\mathrm{J} 1256-0547$ & $2.96 \pm 0.3$ & $-0.579 \pm 0.005$ & $0.051 \pm 0.002$ & $5.7 \pm 3$ & $0.186 \pm 0.02$ & -1508.8 & 3 & 1 \\
\hline $\mathrm{J} 1331+3030$ & $.275 \pm 0.03$ & -1.0 & 9 & .1 & .1 & -50.3 & 01 & 20 \\
\hline J1337-1257 & $1.42 \pm 0.1$ & $-0.621 \pm 0.006$ & 0.053 & 1.55 & 0.190 & -1025.3 & $1.0 \mathrm{E}-02$ & 4 \\
\hline $\mathrm{J} 1427-4206$ & $1.37 \pm 0.1$ & $-0.558 \pm 0.004$ & $0.044 \pm 0.002$ & $0.81 \pm 0.3$ & $0.411 \pm 0.03$ & -1356.1 & 7.3E-02 & 7 \\
\hline $\mathrm{J} 1517-2422$ & $0.741 \pm 0.08$ & $-0.271 \pm 0.005$ & $0.046 \pm 0.002$ & $0.67 \pm 0.2$ & $0.320 \pm 0.02$ & -1150.6 & $9.7 \mathrm{E}-01$ & 9 \\
\hline $\mathrm{J} 1550+0527$ & $0.029 \pm 0.01$ & $-0.701 \pm 0.005$ & $0.045 \pm 0.001$ & $0.243 \pm 0.0$ & $0.115 \pm 0.01$ & -1523.7 & $6.3 \mathrm{E}-02$ & 24 \\
\hline J1617-5848 & $0.24 \pm 0.2$ & $-0.891 \pm 0.004$ & $0.044 \pm 0.001$ & $3.8 \pm 2$ & $0.136 \pm 0.01$ & -1571.7 & $1.1 \mathrm{E}-02$ & 1 \\
\hline J1642+3948 & $1.214 \pm 0.05$ & $-0.749 \pm 0.008$ & $0.059 \pm 0.002$ & $0.79 \pm 0.2$ & $0.166 \pm 0.02$ & -719.0 & 7.3E-01 & 8 \\
\hline J1733-1304 & $0.969 \pm 0.04$ & $-0.670 \pm 0.005$ & $0.049 \pm 0.002$ & $0.56 \pm 0.2$ & $0.180 \pm 0.02$ & -1356.2 & 1.1E-01 & 11 \\
\hline $\mathrm{J} 1751+0939$ & $1.034 \pm 0.08$ & $-0.476 \pm 0.005$ & $0.049=$ & & 0.589 & -957.7 & 02 & 16 \\
\hline J1924-2914 & $1.724 \pm 0.04$ & $-0.643 \pm 0.004$ & $0.044 \pm 0.001$ & $0.36 \pm 0.1$ & $0.210 \pm 0.02$ & -2063.6 & $4.5 \mathrm{E}-03$ & 13 \\
\hline J2000-1748 & $0.63 \pm 0.2$ & $-0.472 \pm 0.009$ & $0.043 \pm 0.004$ & $0.87 \pm 0.4$ & $0.71 \pm 0.1$ & -260.0 & $1.6 \mathrm{E}-01$ & 7 \\
\hline $\mathrm{J} 2025+3343$ & $0.49 \pm 0.1$ & $-0.770 \pm 0.005$ & $0.046 \pm 0.002$ & $0.84 \pm 0.3$ & $0.336 \pm 0.03$ & -1224.1 & 1.7E-01 & 5 \\
\hline J2056-4714 & $0.223 \pm 0.06$ & $-0.634 \pm 0.004$ & $0.043 \pm 0.001$ & $0.56 \pm 0.1$ & $0.253 \pm 0.02$ & -1519.7 & & 11 \\
\hline $\mathrm{J} 2148+0657$ & $0.722 \pm 0.07$ & $-0.971 \pm 0.005$ & $0.052 \pm 0.002$ & $1.45 \pm 0.5$ & $0.139 \pm 0.01$ & -1511.4 & $1.8 \mathrm{E}-01$ & 4 \\
\hline $\mathrm{J} 2232+1143$ & $1.302 \pm 0.08$ & $-0.411 \pm 0.01$ & $0.102 \pm 0.004$ & $0.346 \pm 0.08$ & $0.404 \pm 0.04$ & -397.2 & $8.9 \mathrm{E}-01$ & 9 \\
\hline $\mathrm{J} 2253+1608$ & $2.647 \pm 0.05$ & $-0.533 \pm 0.009$ & $0.072 \pm 0.003$ & $0.48 \pm 0.1$ & $0.217 \pm 0.02$ & -628.8 & 7.4E-01 & 12 \\
\hline $\mathrm{J} 2258-2758$ & $0.83 \pm 0.1$ & $-0.665 \pm 0.006$ & $0.063 \pm 0.002$ & $1.05 \pm 0.2$ & $0.243 \pm 0.02$ & -1541.3 & $4.8 \mathrm{E}-02$ & 6 \\
\hline $\mathrm{J} 2357-5311$ & $-0.002 \pm 0.03$ & $-0.794 \pm 0.004$ & $0.041 \pm 0.001$ & $0.539 \pm 0.1$ & $0.136 \pm 0.01$ & -2259.7 & $1.7 \mathrm{E}-01$ & 11 \\
\hline
\end{tabular}

a "ln Jy" is not a unit, but is indicated to emphasize the definition given in (4). 
$\chi_{n}$ ACF for J0635-7516

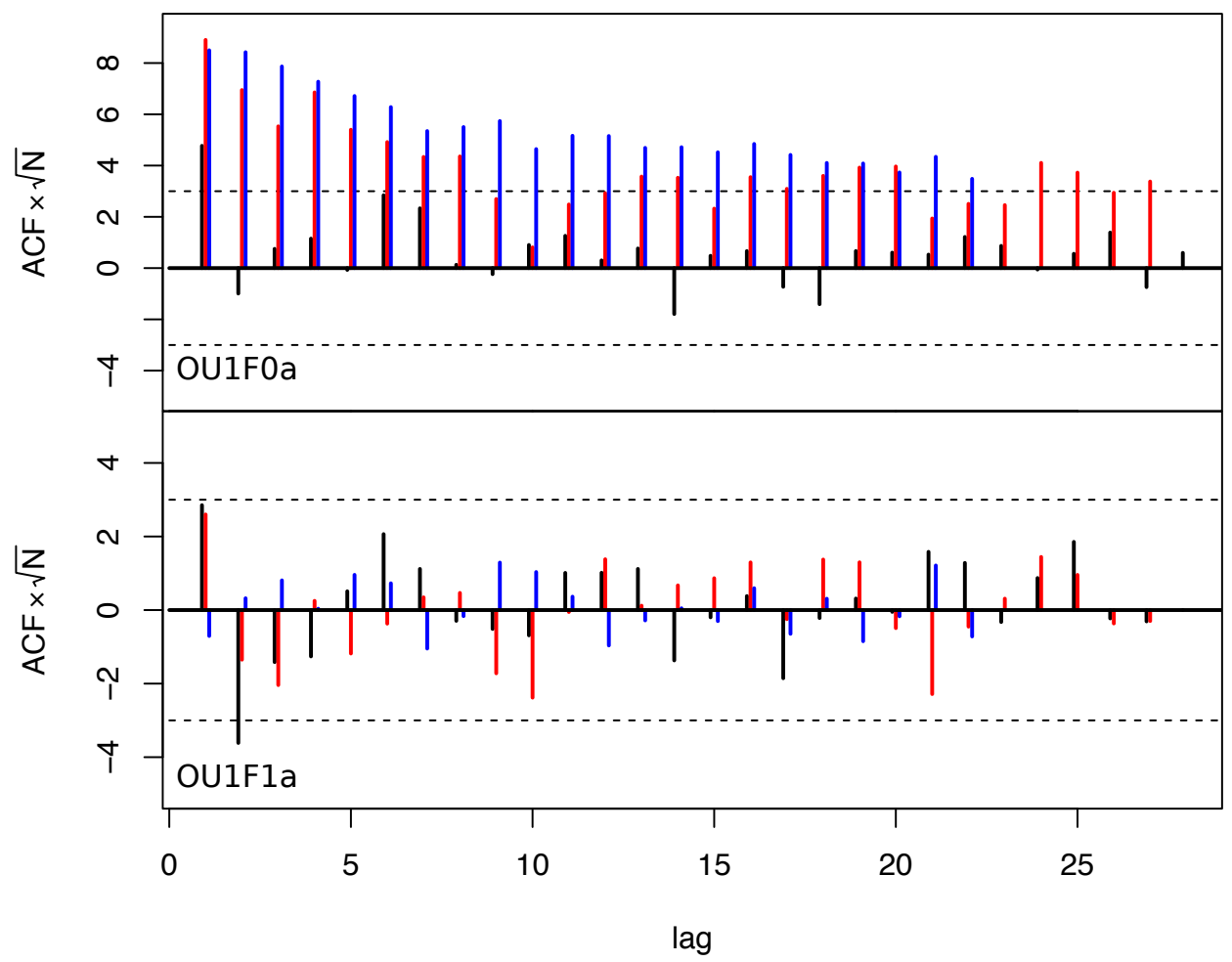

Figure 7. Red, blue, and black bars show the standardized $(\times \sqrt{N})$ ACF of the normalized residuals at Band 3, 7, and all data, respectively. Dashed lines mark the $\pm 3-\sigma$ level assuming no cross-correlation above zero lag. Top panel. Standardized ACF of the OU-1F0a model, parameters in Table 2. Bottom panel. The same for the OU-1F1a model with curvature and $\nu$-lag (Table 4$)$.

Table 3. Comparison of different models applied to J0635-7516.

\begin{tabular}{|c|c|c|c|c|c|}
\hline OU-mix & $\begin{array}{l}\text { Model } \\
\text { Curvature }\end{array}$ & $\nu$-lag & $\Delta \mathrm{AIC}^{a}$ & $\mathrm{p}_{\mathrm{A}-\mathrm{D}}$ & $\mathrm{p}_{\mathrm{L}-\mathrm{B}}^{b}$ \\
\hline $1 \mathrm{~F} 0 \mathrm{a}$ & & & 0 & $2.8 \mathrm{E}-01$ & $2.2 \mathrm{E}-04$ \\
\hline $2 \mathrm{~F} 0 \mathrm{a}$ & & & -58.4 & $5.8 \mathrm{E}-01$ & $2.3 \mathrm{E}-08$ \\
\hline $1 \mathrm{~F} 1 \mathrm{a}$ & & & -335.4 & $6.5 \mathrm{E}-02$ & $1.4 \mathrm{E}-02$ \\
\hline $1 \mathrm{~F} 1 \mathrm{a}$ & $\checkmark$ & & -421.8 & $4.0 \mathrm{E}-02$ & $6.4 \mathrm{E}-06$ \\
\hline $1 \mathrm{~F} 1 \mathrm{a}$ & & $\checkmark$ & -333.5 & $2.8 \mathrm{E}-02$ & $8.2 \mathrm{E}-02$ \\
\hline $1 \mathrm{~F} 1 \mathrm{a}$ & $\checkmark$ & $\checkmark$ & -419.8 & $1.1 \mathrm{E}-01$ & $4.0 \mathrm{E}-03$ \\
\hline $2 \mathrm{~F} 1 \mathrm{a}$ & & & -331.4 & $6.5 \mathrm{E}-02$ & $1.4 \mathrm{E}-02$ \\
\hline
\end{tabular}

${ }^{a}$ Difference with respect to the OU-1F0a AIC given in Table 2.

${ }^{b}$ We use lag $=20$ except for $\mathrm{J} 1331+3030$, for which we use lag $=16$. 
left panels, while the right panels show the normalized residuals $\left(\chi_{n}\right)$ time series and distribution histogram. Less band-systematics in the residuals time series are evident when comparing them with those of Figure 6.

This same analysis - that is, examination of the residuals quality, systematics, biases, and the final values of the AIC and p-values of statistical tests - is applied to the rest of the grid calibrators, allowing us to pick the optimal model for each lightcurve. Hereafter, we refer to these as the "selected models." Table 4 shows the MLE parameters for all selected models. More detailed MLE results including plots as those of Figures 7 and 8 for the rest of the grid calibrators are presented in the supplementary material (Appendix C).

We find that, by a similar evaluation as the one performed on J0635-7516, the selected model includes a spectral index variation term ( $\beta$-term) for all grid calibrators, except J1127-1857. Additionally, a negative curvature parameter $\kappa$ is included in all selected models except that of J1127-1857 and J1331+3030. These sources have the least number of measurements, which impedes us to fit reliably more sophisticated models. The inclusion of $\nu$-lag $(\epsilon)$ in the fitting does improve in 15 cases the quality of the residuals, that is, their $\mathrm{ACF}$ and statistical tests indicate they are more consistent with GWN than the residuals of the model without $\nu$-lag. However, as it can be deduced from the commonly large error bars of $\epsilon$ in Table 4, the $\nu$-lag inclusion does not affect much the likelihood value. As described in Section 3.4, we take a more conservative approach and consider that the actual uncertainty of $\epsilon$ is bounded from below by $0.5 \mathrm{~d}$. Considering this, there are only five sources with estimated $\epsilon$ magnitudes larger than $1.5 \mathrm{~d}$, four of them with $\epsilon<0$.

Only for J2148+0657 we find an $\epsilon$ MLE which appears significantly positive. It is difficult to evaluate in detail why this source displays such a feature, and even more difficult to explain it physically. We note, however, that Band 6 data for this source does appear to be overestimated by the model, and that the remotion of these data produces a MLE for $\epsilon$ which is positive but smaller $(\sim 0.5 \mathrm{~d})$ and only marginally significant. This example indicates us that, while in the ideal case (Section 3.4) and under all the hypotheses (e.g., normality, independence, spectral shape, etc...) we can recover a solid $\nu$-lag as small of 1-2 d, divergences from the ideal case can bias the MLE estimation towards an artificial lag. 

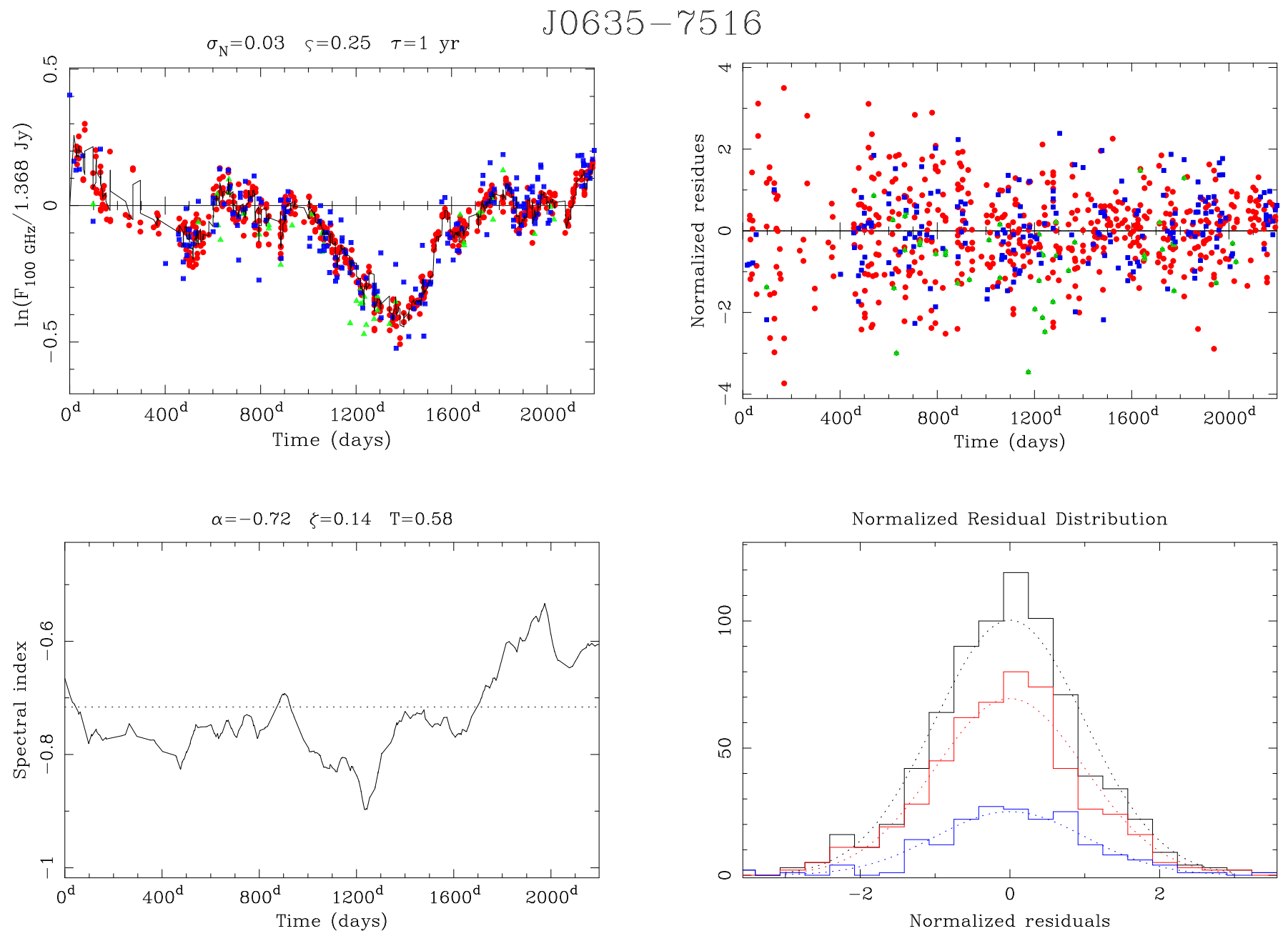

Figure 8. Best-fit OU-1F1a model applied to J0635-7516 with curvature and lag. MLE parameters are given in Table 4. Symbol definition and colors as in Figure 6. Top-left. Centered data and predictions. Top-right. $\chi_{n}$ time series. Bottom-left. Local spectral index time series at $100 \mathrm{GHz}$ (15). Bottom-right. Residuals histogram. 
Table 4: MLE parameters of the selected OU model for each source.

\begin{tabular}{|c|c|c|c|c|c|c|c|c|c|c|}
\hline Source & $\mu$ & $\alpha$ & $\kappa$ & $\begin{array}{c}\epsilon \\
(\mathrm{d})\end{array}$ & $\sigma_{N, 0}$ & $\left(\mathrm{yr}^{-1 / 2}\right)$ & $\left(\mathrm{yr}^{-1 / 2}\right)$ & $\begin{array}{l}\tau_{1} \\
(\mathrm{yr})\end{array}$ & $\begin{array}{c}T \\
(\mathrm{yr})\end{array}$ & $\rho$ \\
\hline J0006-0623 & $0.97 \pm 0.3$ & $-0.326 \pm 0.04$ & $-0.191 \pm 0.01$ & - & $0.017 \pm 0.0008$ & $0.221 \pm 0.02$ & $0.152 \pm 0.02$ & $5.4 \pm 5$ & $0.61 \pm 0.2$ & $0.66 \pm 0.1$ \\
\hline $\mathrm{J} 0237+2848$ & $0.781 \pm 0.06$ & $-0.449 \pm 0.02$ & $-0.147 \pm 0.01$ & - & $0.017 \pm 0.001$ & $0.316 \pm 0.02$ & $0.324 \pm 0.04$ & $0.52 \pm 0.2$ & $0.091 \pm 0.03$ & $0.44 \pm 0.1$ \\
\hline J0238+1636 & $0.39 \pm 0.2$ & $-0.307 \pm 0.02$ & $-0.145 \pm 0.01$ & $-0.15 \pm 0.2$ & $0.020 \pm 0.001$ & $0.540 \pm 0.03$ & $0.419 \pm 0.08$ & $0.79 \pm 0.3$ & $0.054 \pm 0.01$ & $0.56 \pm 0.1$ \\
\hline $\mathrm{J} 0319+4130$ & $2.80 \pm 0.1$ & $-0.561 \pm 0.02$ & $-0.131 \pm 0.02$ & - & $0.017 \pm 0.001$ & $0.166 \pm 0.02$ & $0.227 \pm 0.08$ & $2.1 \pm 1$ & $0.065 \pm 0.04$ & $0.03 \pm 0.2$ \\
\hline J0334-4008 & $-0.2 \pm 1$ & $-0.496 \pm 0.02$ & $-0.106 \pm 0.01$ & $0.67 \pm 0.2$ & $0.019 \pm 0.001$ & $0.355 \pm 0.02$ & $0.263 \pm 0.04$ & $19.8 \pm 20$ & $0.128 \pm 0.05$ & $0.56 \pm 0.1$ \\
\hline J0423-0120 & $1.16 \pm 0.7$ & $-0.328 \pm 0.03$ & $-0.218 \pm 0.02$ & - & $0.025 \pm 0.001$ & $0.410 \pm 0.03$ & $0.286 \pm 0.04$ & $7.2 \pm 8$ & $0.248 \pm 0.07$ & $0.60 \pm 0.1$ \\
\hline $\mathrm{J} 0510+1800$ & $0.91 \pm 0.2$ & $-0.193 \pm 0.02$ & $-0.185 \pm 0.01$ & - & $0.020 \pm 0.001$ & $0.520 \pm 0.03$ & $0.292 \pm 0.04$ & $0.82 \pm 0.4$ & $0.144 \pm 0.06$ & $0.43 \pm 0.1$ \\
\hline J0519-4546 & $0.279 \pm 0.07$ & $-0.197 \pm 0.02$ & $-0.167 \pm 0.01$ & - & $0.018 \pm 0.0009$ & $0.376 \pm 0.03$ & $0.382 \pm 0.04$ & $0.46 \pm 0.2$ & $0.093 \pm 0.03$ & $0.09 \pm 0.1$ \\
\hline J0522-3627 & $1.737 \pm 0.06$ & $-0.043 \pm 0.02$ & $-0.167 \pm 0.02$ & $-1.79 \pm 0.2$ & $0.017 \pm 0.001$ & $0.567 \pm 0.03$ & $0.342 \pm 0.08$ & $0.242 \pm 0.07$ & $0.059 \pm 0.02$ & $0.51 \pm 0.1$ \\
\hline J0538-4405 & $0.92 \pm 0.5$ & $-0.437 \pm 0.02$ & $-0.115 \pm 0.01$ & - & $0.021 \pm 0.0009$ & $0.309 \pm 0.02$ & $0.165 \pm 0.02$ & $7.8 \pm 8$ & $0.303 \pm 0.08$ & $0.736 \pm 0.09$ \\
\hline J0635-7516 & $0.31 \pm 0.1$ & $-0.716 \pm 0.04$ & $-0.175 \pm 0.02$ & $0.1 \pm 1$ & $0.035 \pm 0.001$ & $0.245 \pm 0.02$ & $0.143 \pm 0.03$ & $1.02 \pm 0.5$ & $0.58 \pm 0.3$ & $0.25 \pm 0.2$ \\
\hline $\mathrm{J} 0750+1231$ & $0.31 \pm 0.1$ & $-0.497 \pm 0.03$ & $-0.146 \pm 0.02$ & - & $0.027 \pm 0.001$ & $0.234 \pm 0.02$ & $0.113 \pm 0.03$ & $1.7 \pm 1$ & $0.40 \pm 0.2$ & $0.39 \pm 0.2$ \\
\hline $\mathrm{J} 0854+2006$ & $1.51 \pm 0.1$ & $-0.217 \pm 0.03$ & $-0.181 \pm 0.02$ & $-0.853 \pm 0.04$ & $0.026 \pm 0.002$ & $0.703 \pm 0.04$ & $0.217 \pm 0.04$ & $0.43 \pm 0.2$ & $0.204 \pm 0.09$ & $0.17 \pm 0.2$ \\
\hline J0904-5735 & $0.07 \pm 0.1$ & $-0.325 \pm 0.05$ & $-0.051 \pm 0.03$ & - & $0.018 \pm 0.003$ & $0.735 \pm 0.08$ & $0.45 \pm 0.1$ & $0.30 \pm 0.2$ & $0.131 \pm 0.09$ & $0.14 \pm 0.3$ \\
\hline J1037-2934 & $0.25 \pm 0.2$ & $-0.322 \pm 0.03$ & $-0.181 \pm 0.02$ & $0.22 \pm 0.4$ & $0.031 \pm 0.002$ & $0.422 \pm 0.03$ & $0.267 \pm 0.04$ & $1.3 \pm 1$ & $0.125 \pm 0.05$ & $0.44 \pm 0.1$ \\
\hline $\mathrm{J} 1058+0133$ & $1.30 \pm 0.2$ & $-0.368 \pm 0.04$ & $-0.121 \pm 0.02$ & $-0.28 \pm 0.7$ & $0.026 \pm 0.002$ & $0.335 \pm 0.03$ & $0.139 \pm 0.03$ & $1.9 \pm 1$ & $0.56 \pm 0.3$ & $0.36 \pm 0.2$ \\
\hline $\mathrm{J} 1107-4449$ & $0.184 \pm 0.06$ & $-0.465 \pm 0.03$ & $-0.236 \pm 0.02$ & - & $0.025 \pm 0.002$ & $0.270 \pm 0.02$ & $0.321 \pm 0.04$ & $0.55 \pm 0.3$ & $0.163 \pm 0.06$ & $0.26 \pm 0.1$ \\
\hline $\mathrm{J} 1127-1857$ & $0.09 \pm 0.1$ & $-0.583 \pm 0.007$ & - & - & $0.024 \pm 0.002$ & $0.280 \pm 0.05$ & - & $1.64 \pm 0.8$ & - & - \\
\hline $\mathrm{J} 1146+3958$ & $0.19 \pm 0.2$ & $-0.287 \pm 0.04$ & $-0.266 \pm 0.02$ & - & $0.022 \pm 0.002$ & $0.495 \pm 0.04$ & $0.519 \pm 0.08$ & $1.08 \pm 0.5$ & $0.106 \pm 0.03$ & $0.55 \pm 0.1$ \\
\hline $\mathrm{J} 1229+0203$ & $2.21 \pm 0.1$ & $-0.472 \pm 0.03$ & $-0.288 \pm 0.02$ & $-3.89 \pm 0.7$ & $0.024 \pm 0.001$ & $0.393 \pm 0.03$ & $0.370 \pm 0.04$ & $1.02 \pm 0.5$ & $0.174 \pm 0.06$ & $0.27 \pm 0.1$ \\
\hline J1256-0547 & $2.78 \pm 0.3$ & $-0.381 \pm 0.03$ & $-0.189 \pm 0.02$ & - & $0.027 \pm 0.001$ & $0.317 \pm 0.02$ & $0.307 \pm 0.08$ & $3.2 \pm 4$ & $0.139 \pm 0.07$ & $0.14 \pm 0.2$ \\
\hline $\mathrm{J} 1331+3030$ & $-0.294 \pm 0.04$ & $-1.079 \pm 0.04$ & - & - & $0.025 \pm 0.005$ & $0.248 \pm 0.09$ & $1.08 \pm 0.9$ & $0.29 \pm 0.2$ & $0.026 \pm 0.06$ & $0.27 \pm 0.6$ \\
\hline $\mathrm{J} 1337-1257$ & $1.38 \pm 0.2$ & $-0.350 \pm 0.03$ & $-0.222 \pm 0.02$ & $-1.667 \pm 0.02$ & $0.026 \pm 0.001$ & $0.267 \pm 0.02$ & $0.215 \pm 0.04$ & $2.8 \pm 2$ & $0.191 \pm 0.06$ & $0.60 \pm 0.1$ \\
\hline $\mathrm{J} 1427-4206$ & $1.34 \pm 0.1$ & $-0.331 \pm 0.02$ & $-0.191 \pm 0.02$ & - & $0.028 \pm 0.001$ & $0.428 \pm 0.03$ & $0.219 \pm 0.08$ & $0.93 \pm 0.4$ & $0.146 \pm 0.08$ & $0.40 \pm 0.1$ \\
\hline $\mathrm{J} 1517-2422$ & $0.82 \pm 0.1$ & $-0.019 \pm 0.03$ & $-0.240 \pm 0.02$ & - & $0.023 \pm 0.001$ & $0.392 \pm 0.02$ & $0.226 \pm 0.04$ & $1.05 \pm 0.5$ & $0.212 \pm 0.09$ & $0.34 \pm 0.1$ \\
\hline $\mathrm{J} 1550+0527$ & $0.031 \pm 0.02$ & $-0.465 \pm 0.04$ & $-0.235 \pm 0.02$ & - & $0.028 \pm 0.001$ & $0.186 \pm 0.02$ & $0.137 \pm 0.03$ & $0.30 \pm 0.1$ & $0.54 \pm 0.3$ & $0.26 \pm 0.2$ \\
\hline $\mathrm{J} 1617-5848$ & $0.30 \pm 0.2$ & $-0.599 \pm 0.03$ & $-0.237 \pm 0.02$ & - & $0.027 \pm 0.001$ & $0.171 \pm 0.02$ & $0.197 \pm 0.04$ & $5.7 \pm 7$ & $0.27 \pm 0.2$ & $0.00 \pm 0.3$ \\
\hline $\mathrm{J} 1642+3948$ & $1.218 \pm 0.09$ & $-0.353 \pm 0.03$ & $-0.343 \pm 0.02$ & - & $0.022 \pm 0.002$ & $0.305 \pm 0.03$ & $0.455 \pm 0.08$ & $0.81 \pm 0.4$ & $0.091 \pm 0.04$ & $0.46 \pm 0.1$ \\
\hline J1733-1304 & $1.000 \pm 0.07$ & $-0.417 \pm 0.03$ & $-0.242 \pm 0.02$ & - & $0.031 \pm 0.001$ & $0.222 \pm 0.02$ & $0.224 \pm 0.04$ & $0.83 \pm 0.4$ & $0.164 \pm 0.06$ & $0.45 \pm 0.1$ \\
\hline $\mathrm{J} 1751+0939$ & $1.08 \pm 0.1$ & $-0.155 \pm 0.03$ & $-0.289 \pm 0.02$ & $-1.56 \pm 0.4$ & $0.020 \pm 0.001$ & $0.685 \pm 0.04$ & $0.272 \pm 0.04$ & $0.39 \pm 0.2$ & $0.136 \pm 0.05$ & $0.37 \pm 0.1$ \\
\hline J1924-2914 & $1.755 \pm 0.08$ & $-0.317 \pm 0.02$ & $-0.289 \pm 0.01$ & $1.00+0$ & $0.028 \pm 0.001$ & $0.238 \pm 0.02$ & $0.281 \pm 0.05$ & $0.82 \pm 0.4$ & $0.094 \pm 0.04$ & $0.42 \pm 0.1$ \\
\hline $\mathrm{J} 2000-1748$ & $0.63 \pm 0.3$ & $-0.335 \pm 0.04$ & $-0.123 \pm 0.03$ & - & $0.018 \pm 0.002$ & $0.495 \pm 0.04$ & $0.324 \pm 0.08$ & $2.0 \pm 1$ & $0.137 \pm 0.07$ & $0.44 \pm 0.2$ \\
\hline $\mathrm{J} 2025+3343$ & $0.52 \pm 0.2$ & $-0.412 \pm 0.03$ & $-0.330 \pm 0.02$ & - & $0.023 \pm 0.001$ & $0.416 \pm 0.03$ & $0.369 \pm 0.05$ & $1.00 \pm 0.4$ & $0.111 \pm 0.04$ & $0.33 \pm 0.1$ \\
\hline $\mathrm{J} 2056-4714$ & $0.238 \pm 0.08$ & $-0.329 \pm 0.02$ & $-0.263 \pm 0.01$ & $1.36 \pm 0.7$ & $0.017 \pm 0.001$ & $0.312 \pm 0.02$ & $0.392 \pm 0.04$ & $0.73 \pm 0.3$ & $0.055 \pm 0.01$ & $0.44 \pm 0.1$ \\
\hline $\mathrm{J} 2148+0657$ & $0.73 \pm 0.1$ & $-0.539 \pm 0.02$ & $-0.383 \pm 0.02$ & $5.99 \pm 0.9$ & $0.021 \pm 0.001$ & $0.191 \pm 0.01$ & $0.603 \pm 0.08$ & $2.0 \pm 1$ & $0.051 \pm 0.01$ & $0.633 \pm 0.09$ \\
\hline $\mathrm{J} 2232+1143$ & $1.31 \pm 0.2$ & $0.086 \pm 0.07$ & $-0.421 \pm 0.01$ & - & $0.018 \pm 0.001$ & $0.598 \pm 0.04$ & $0.525 \pm 0.06$ & $0.86 \pm 0.7$ & $0.25 \pm 0.1$ & $0.21 \pm 0.1$ \\
\hline $\mathrm{J} 2253+1608$ & $2.64 \pm 0.1$ & $-0.176 \pm 0.03$ & $-0.303 \pm 0.02$ & $-0.3 \pm 1$ & $0.018 \pm 0.001$ & $0.301 \pm 0.02$ & $0.331 \pm 0.04$ & $1.01 \pm 0.5$ & $0.198 \pm 0.07$ & $0.56 \pm 0.1$ \\
\hline $\mathrm{J} 2258-2758$ & $0.96 \pm 0.3$ & $-0.388 \pm 0.03$ & $-0.246 \pm 0.01$ & $0.14 \pm 0.2$ & $0.021 \pm 0.0009$ & $0.349 \pm 0.02$ & $0.258 \pm 0.03$ & $2.28 \pm 0.7$ & $0.286 \pm 0.08$ & $0.703 \pm 0.07$ \\
\hline $\mathrm{J} 2357-5311$ & $0.052 \pm 0.08$ & $-0.524 \pm 0.03$ & $-0.211 \pm 0.01$ & $0.18 \pm 0.7$ & $0.023 \pm 0.0008$ & $0.179 \pm 0.02$ & $0.107 \pm 0.02$ & $1.26 \pm 0.5$ & $0.65 \pm 0.4$ & $0.53 \pm 0.1$ \\
\hline
\end{tabular}




\subsection{Forecasts and uncertainty intervals.}

Using the MLE parameters and the model, we can derive flux forecasts and its stochastic uncertainties for each source using the Kalman recursions (Appendix B) and the parametric uncertainty (see Section 3.3). In the characteristic monitoring time scale of the grid calibrators, the main source of uncertainty is given by the intrinsic time variability. In the long-term $(t>\tau, T)$, the parametric uncertainty of the deterministic model usually dominates.

Figure 9 shows the five following measurements of J0635-7516 in Bands 3 and 7, which occur during a time interval of $\sim 60$ days after the last measurement considered in this work, taken in 2018-07-07. Panels (a) and (c) also shows the forecasts from the OU$1 F 0 a$ and the selected OU-1F1a model during this time, together with the evolving $\pm 1 \sigma$ uncertainty intervals. These intervals include the parametric and stochastic variability, added in quadrature. Panels (b) and (d) display the breakdown of the uncertainty in its components (Section 3.3). Figure 9 shows predictions based only on the data taken up to 2018-07-07 for reference, but in practice it would be desirable to update the model at each new measurement. Figure 9 shows that most measurements fall within the $\pm 1 \sigma$ uncertainty interval around the forecasts in both models, with all measurements falling within $\pm 2 \sigma$. Note that the selected model OU-1F1a performs better than the OU-1F0a model, specially in Band 7.

We compare the 91.5 and $343.5 \mathrm{GHz}$ flux forecasts for the rest of the grid calibrators with their fluxes measured immediately after the last observed time given in Table 1. For the full grid sample, there are 56 and 41 of these measurements in total for Bands 3 and 7, respectively. According to the hypotheses, the standardized differences between the fluxes and forecasts should be distributed as a standard Gaussian. An AndersonDarling test on both Band 3 and 7 standardized differences indicate that we cannot reject the Gaussian hypothesis at the $95 \%$ confidence level in either case. We test the null hypothesis further by using two more tests. We calculate the mean of the standardized differences and the sum of its squares. According to the null hypotheses, they should distribute as Gaussian with standard deviation $n^{-1 / 2}$ and a $\chi_{n}^{2}$ distribution, respectively, where $n$ is the number of measurements. For Band 3, we find that the standardized differences are consistent with the null hypotheses in both tests. This is also the case for the means of the Band 7 means standardized differences. However, the sum of its squares is 20.6, while the $95 \%$ (two-sided) interval of the $\chi_{41}^{2}$ distribution starts at 25.22. Therefore, it is possible that the uncertainties of the Band 7 forecasts are overestimated

by a factor $\sqrt{25.22 / 20.6}$, or an $11 \%$. An overestimation of this type - meaning that the forecasts are actually closer to the measured values than expected - would affect the reported flux uncertainties, and it may decrease the sensitivity of the model to detect outliers. It would also overestimate the interpolated flux calibration error, either in a science project or during the quality evaluation of new data for ingestion to the source catalog.

Finally, we evaluate the accuracy of the parametric uncertainty estimation given 

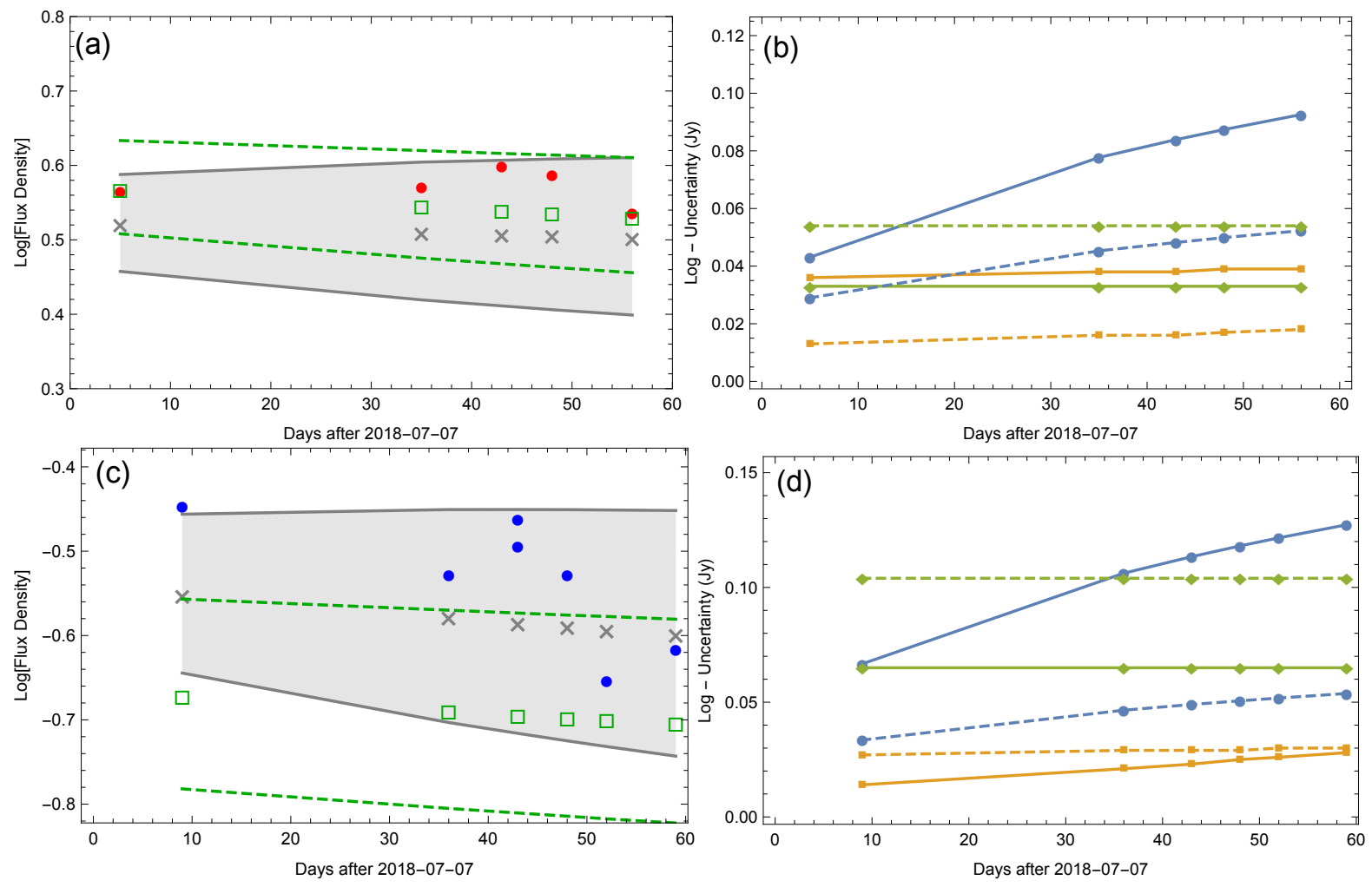

Figure 9. Stochastic model forecast of J0635-7516 during the 60 days ensuing the last measurement used for the analysis of this work. (a). Red dots: $91.5 \mathrm{GHz}$ fluxes. Crosses and squares show the forecasts of the OU-1F1a ( $\nu$-lag + curvature) and the OU$1 \mathrm{~F} 0$ a model, respectively. Forecasts are bracketed by the $\pm 1 \sigma$ total uncertainty interval (parametric+stochastic) shown in grey continuous and dashed green lines for the OU1F1a (lag+curvature) and the OU-1F0a model, respectively. (b). Uncertainties of the 91.5 GHz flux. In blue (dots), orange (squares), and green (diamonds) the stochastic, parametric uncertainty, and measurement uncertainty levels, respectively. Continuous and dashed lines indicate the results of the OU-1F1a (lag+curvature) and the OU-1F0a model, respectively. (c). Same as panel (a), but with blue dots showing fluxes at 343.5 GHz. (d). Same as panel (b) but at $343.5 \mathrm{GHz}$.

by Equation (19) using Monte Carlo simulations. We use a bayesian model to estimate the parametric uncertainty in the general case when the likelihood function is not well approximated by a normal near the maximum - as it happens for some parameters in J0635-7516. This source is representative of those calibrators in which the monitoring time is not sufficiently long in order to obtain an accurate estimation of the decorrelation time. According to the bayesian prescription, the posterior probability distribution of the parameters is given by the likelihood times the prior. We choose the latter to be minimally informative, that is, a constant greater than zero in the allowed parameter space. The allowed parameter space includes only the positive values for those parameters expected to be positive (e.g., decorrelation times and variance rates) and it is unconstrained otherwise. We sample the posterior using a Metropolis-Hastings Markov chain (Gregory 2005) starting from the MLE, and calculate the forecasts for all 
the simulated parameters. This sampling should take into account all the non-linearities involved in the calculation of the deterministic model and in deriving a forecast.

We find that the estimations of the parametric uncertainty derived from (19) are comparable, although usually larger by a factor between 1-3 times the standard deviation of the forecasts sampled from the posterior distribution. That is, the formulae derived in Section 3.3 gives a conservative estimation of the parametric uncertainty. Because usually this parametric uncertainty does not dominate the forecast uncertainties in the short term, we use (19) to estimate it for simplicity.

4.2.1. Flux interpolation: comparison between two methods. Because the stochastic model includes a description of the time variability and the spectral characteristics of the source, we can produce forecasts and interpolations of the flux at any time and frequency. These interpolations and forecasts have uncertainties derived from the variability of the source and from the measurement error.

Another way (similar to the one used, for example, in the ALMA pipeline up to Cycle 5\|) to interpolate (and extrapolate) the flux from the source catalog uses the closest (in time) Band 3 measurement and a spectral index derived from the closest pair of measurements - Bands $3 \& 6$ or $3 \& 7$ - taken on the same day. Using this Band 3 flux and spectral index, the expected flux density at other frequencies are calculated. We will refer to this method as P0. The error of this flux is derived from the uncertainty of each flux measurement, but there is no estimation of the uncertainty derived from the variability of the source.

We compare both methods of interpolating the flux on J0635-7516 by ignoring one day of observation and obtaining the interpolated values for this day using the stochastic model fitted to the source (OU-1F1a) and P0. We repeat this process for each day, and compare these interpolations with the fluxes actually measured. Figure 10 shows histograms of the percentage difference between the measured data and the P0 and stochastic model interpolations. The histograms make clear that the stochastic model differences respect to the measured data are smaller than those provided by $\mathrm{P} 0$. For P0, the standard deviation of the differences at Bands 3 and 7 are $7 \%$ and 11\%, respectively, while for the OU-1F1a interpolations, these are $3 \%$ and $5 \%$, respectively.

|| Pipeline documentation: https://almascience.org/documents-and-tools/processing/science-pipeline. The specific task used to obtain interpolated fluxes from the source catalog is getALMAFlux, described in https://safe.nrao.edu/wiki/bin/view/ALMA/GetALMAFlux

ๆ These numbers are not to be confused with the typical flux uncertainty of ALMA. 


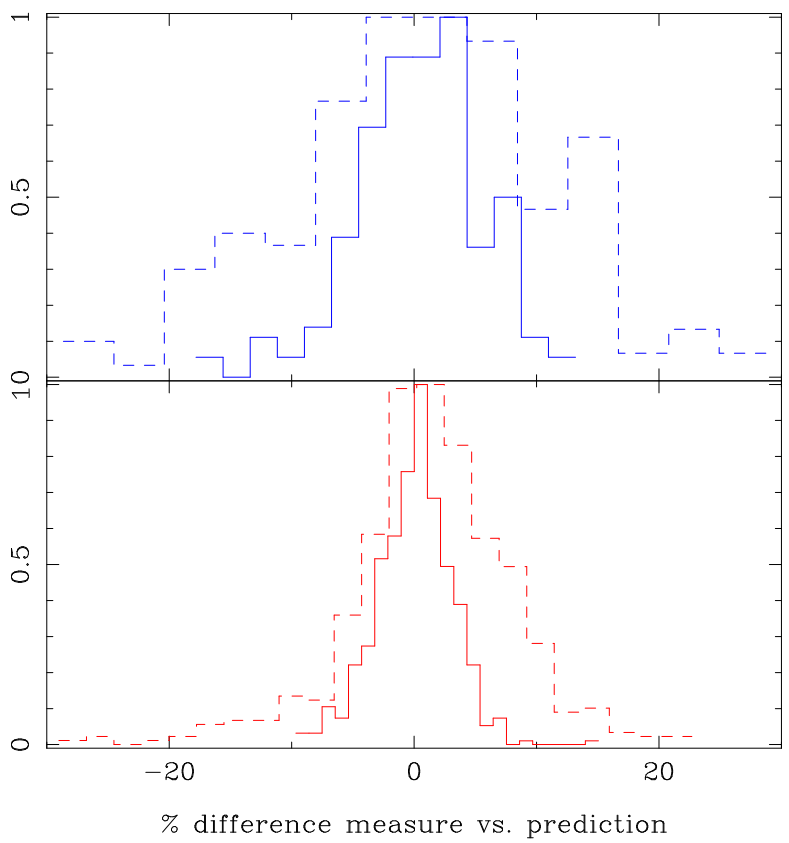

Figure 10. Bottom and top panels show, respectively, the Band 3 and 7 normalized histograms of the percentage difference between the measured flux density and the P0 interpolation (dashed lines) and stochastic model interpolation (continuous line).

\section{Discussion}

In this section we show in more detail some additional applications and extensions of the stochastic modeling (Sections 5.1. and 5.2) and evaluate some of its drawbacks and future improvements (Section 5.3).

Stochastic variability studies of extragalactic sources have commonly focused on accurately determining the parameters of the process in order to link them with the underlying physics. The use of the stochastic process to make forecasts and to interpolate the flux at unobserved times has received less attention in astronomy. Areas of the ALMA operations where the work presented in this paper is of practical use are: (i) the monitoring of grid calibrators and (ii) science data reduction and the quality assurance process.

During the calibrator survey monitoring of the grid sources, it is important to compare each new measurement with the previous history of the calibrator. The stochastic model provides updated forecasts of the measurement at the new observing times and associated uncertainties based on the observed variability of the source (as shown in Section 4.2). Both these quantities allow the calibration JAO group to evaluate precisely how consistent is the new measurement with the historical variations of the quasar (and identify outliers and flaring behavior).

Regarding possible applications to data reduction, note that the flux of the secondary flux calibrator for each science project is determined from an interpolation (in time and frequency) based on previous measurements obtained by the calibrator survey. 
The stochastic model allow us to calculate this interpolated flux and the associated (variability induced) uncertainty. Furthermore, in a similar fashion compared with the calibrator survey, the stochastic modeling of the amplitude/phase calibrator allows us to evaluate quantitatively whether its derived flux is consistent with the previous catalogue values. This assessment of the reliability and uncertainty of the flux calibration is commonly done during the quality assurance process. Section 5.1 shows examples of stochastic modeling on amplitude/phase (non-grid) calibrators.

\subsection{Application to less frequently sampled data: phase calibrators}

In principle, the methods presented in this study can be applied to the light-curve of any quasar in $\mathrm{mm} / \mathrm{sub}-\mathrm{mm}$ wavelengths, including the rest of the calibrators in the ALMA source catalog. Although the present study is focused on the grid sources, this is mainly because they have more measurements compared to the rest of the calibrators. The scant data on some calibrators increases greatly the parameter degeneracy and uncertainty.

To illustrate the applicability of these methods to phase calibrators not in the grid source list, we select the subset of ten best sampled sources in the ALMA source catalog (Table 5). Because of the smaller number of points, in order to remove some parameter degeneracy, we used a OU-1F1a model without curvature and with two additional constrains: $\sigma_{N, 0}=0.06$ fixed and we took equal decorrelation timescales for the flux and spectral indices $(T=\tau)$. Figures showing the results of the fitting for all these sources are presented in the supplementary material.

In a typical ALMA science project, the flux determined for the phase calibrator is examined in order to determine the plausibility of the flux calibration. If the flux is not consistent with the observed variability, this may indicate problems with the data reduction or calibration. This is one of the basic tests performed during the quality assurance stage of each ALMA project, and a common practice in the radio-astronomy community. However, determining what is a flux consistent with the variability of the phase calibrator is not usually well defined. The dispersion of the values of the light-curve may serve as a first approximation. However, intuitively, this dispersion is certainly too large if the phase calibrator flux was measured (that is, absolutely calibrated against a primary flux calibrator) not long before or after the science project in question. The present work defines quantitative uncertainties on these fluxes taking into consideration the historical variability of the source, and therefore defines precisely how consistent is the new measurement with those in the catalog.

In Table 5 we compare the interpolations derived from the P0 method (see Section 4.2.1) and those derived from the stochastic modeling for the ten best sampled phase calibrators. We calculate the median (per source and Band) of the absolute value of the relative differences (in percentage) between the interpolated fluxes calculated using both methods and the actual measurements (given by Bonato et al. 2018). We assume the fluxes given by Bonato et al. (2018) are independent from the measurements of the phase calibrators given in the ALMA source catalog. We can see that in the 
Table 5. Median of the absolute differences in percentage between the fluxes given by Bonato et al. (2018) and those extra- and interpolated from the ALMA source catalog. For each entry, the first number is associated with the P0 flux estimation algorithm, and the second uses the prediction based on a OU-1F1a model fitted to each source.

\begin{tabular}{lrcccccc}
\hline Source & $\#$ & \multicolumn{6}{c}{ Band } \\
& meas. & \multicolumn{1}{c}{3} & 4 & 6 & 7 & 8 & 9 \\
\hline J0106-4034 & 14 & $3.7,2.9$ & $\ldots$ & $72.9,60.1$ & $52.6,1.9$ & $\ldots$ & $\ldots$ \\
J0132-1654 & 28 & $\ldots$ & $\ldots$ & $25.0,66.0$ & $26.7,6.4$ & $\ldots$ & $\ldots$ \\
J0217+0144 & 42 & $13.4,7.4$ & $\ldots$ & $9.2,12.2$ & $11.0,14.7$ & $30.5,28.5$ & $15.0,18.4$ \\
J0601-7036 & 42 & $8.1,4.9$ & $\ldots$ & $11.1,8.8$ & $8.4,1.4$ & $25.8,32.6$ & $66.9,69.0$ \\
J0607-0834 & 48 & $7.5,5.4$ & $8.1,11.0$ & $8.3,7.0$ & $7.8,7.3$ & $14.5,12.8$ & $14.6,15.3$ \\
J1626-2951 & 33 & $22.1,7.8$ & $20.5,6.6$ & $23.9,25.0$ & $17.3,18.6$ & $\ldots$ & $42.4,31.6$ \\
J1744-3116 & 42 & $15.0,10.7$ & $10.2,3.8$ & $9.5,12.3$ & $10.2,18.5$ & $41.6,8.7$ & $\ldots$ \\
J2134-0153 & 186 & $10.8,10.0$ & $\ldots$ & $6.3,6.6$ & $4.2,3.6$ & $\ldots$ & $61.8,60.8$ \\
J2157-6941 & 16 & $21.5,38.2$ & $\ldots$ & $\ldots$ & $48.6,13.3$ & $\ldots$ & $\ldots$ \\
J2225-0457 & 29 & $11.7,5.1$ & $\ldots$ & $\ldots$ & $26.0,22.0$ & $\ldots$ & $57.7,43.8$ \\
\hline
\end{tabular}

majority of the cases (26 of 40, among all Bands and sources) the median of the absolute differences is lower for the stochastic estimations. We confirm the significance of this result using a binomial distribution test, which reject the hypothesis that both methods are equivalent (and favors the stochastic interpolation residuals being lower) at a $95 \%$ confidence level. Furthermore, we cannot confirm that the P0 flux interpolation is significantly (p-value lower than 0.05 ) better than the stochastic method in any band.

Figure 11 shows, for J0217+0144 as a representative example, the data from the source catalog (filled circles), from Bonato et al. (2018) (open circles), the interpolation and extrapolations of the stochastic modeling (stars) and those of the method described above (squares). The error bars are centered in the stochastic model values, and display the uncertainty of the flux including the variability of the source. Note additionally that the first "prediction" of the model is rather a retrodiction (or hindcast) because there is no previous data on the source. It is equivalent to a forecast due to the time reversibility of the stochastic process. 


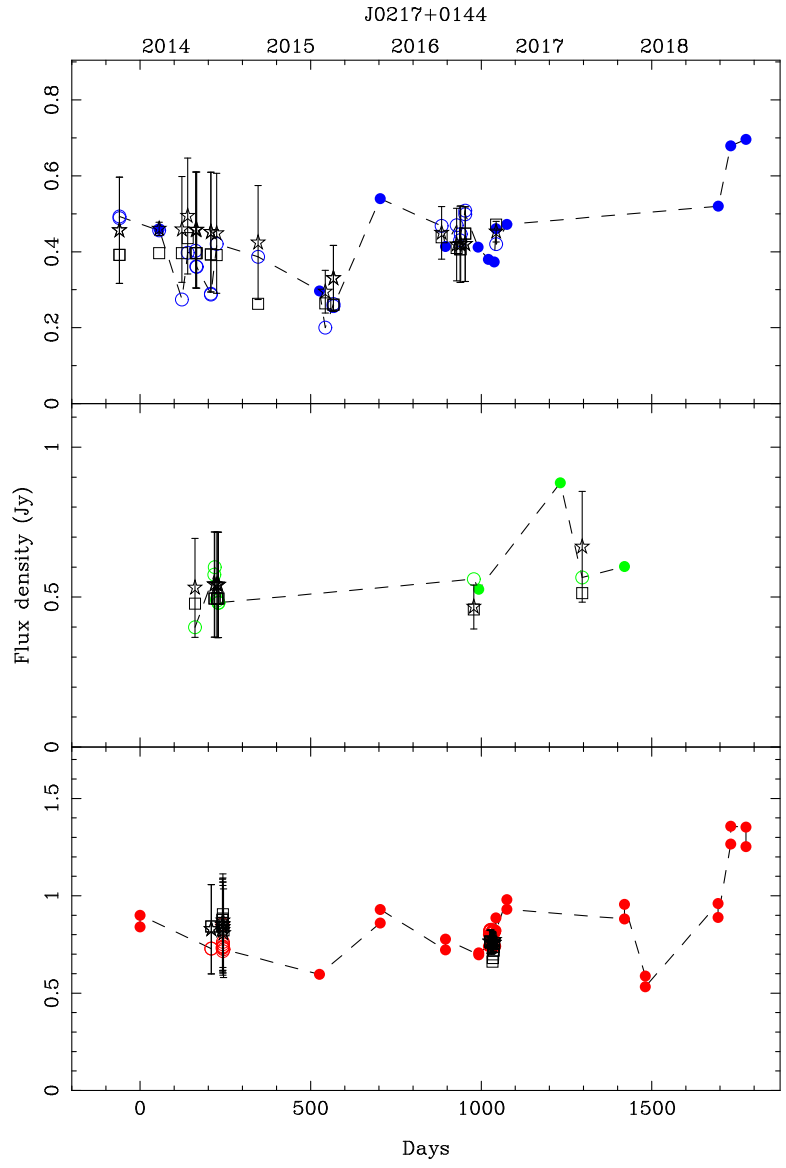

Figure 11. Top panel: Band 7 data of J0217+0144. Blue filled and open circles show the source catalog and Bonato et al. (2018) fluxes, respectively. Inter- and extrapolations from the stochastic model are marked with stars, and those from P0 are marked by squares. Error bars centered on the stochastic interpolations show their expected uncertainty. Middle panel: same as above but in Band 6 (green). Bottom panel: same as top panel but in Band 3 data (red).

\subsection{Combining calibrator survey with additional data.}

The flux densities used in the light-curves analyzed in this work come from data taken by the calibration survey and reported in the source catalog. Additional measurements for many calibrators are provided by the ALMACAL survey (Oteo et al. 2016, Bonato et al. 2018). In principle, any other sub-mm measurements can be used in conjunction with the source catalog to model the light-curves, as long as the new data: (i) have comparable measurement errors as the source catalog; and (ii) the new data's calibration is independent from the source catalog. Regarding (i), because the main source of measurement error comes from calibration, there are no large differences between the ALMACAL flux uncertainties and those of the source catalog. It is also desirable to combine datasets with similar characteristics and comparable cadency and time coverage in order to obtain a robust stochastic fitting. Requirement (i) may be circumvented in the future using a more sophisticated heteroscedasticity prescription. Regarding point (ii), while most ALMACAL data is calibrated independently from the grid source 
catalog, not all of it is. Therefore, we emphasize this section having the purpose of showing how the stochastic model can be extended to data taken with a larger frequency spread, rather than proposing a more complete model or a procedure applicable to all the grid sources. In the following and for the rest of this section, therefore, we treat requirement (ii) as an assumption.

Because the additional ALMACAL data on source J0635-7516 do not include Bands above 7, for this section testing we choose J2253+1608 (3C 454.3), which has been used as a high-frequency calibrator and has been observed much more frequently in Bands 8 to 10. Figure 12 shows the light-curve and spectrum of this source including the additional data. We eliminate one Band 3 measurement from the combined dataset as an outlier because it is $60 \%$ larger than the source catalog flux. Again, we try to qualify data as outlier very sparingly.

Figures 13 and 14 shows the results of the OU-1F1a model fitted to J2253+1608, to the source catalog data only and the combined dataset, respectively. The models shown in Figures 13 and 14 are similar in general, but there are some noticeable differences in the long-term spectrum. This is illustrated in the right panel of Figure 12: Band 8 and 9 data indicate that the model fitted to the data between Bands 3 and 7 has too a pronounced concavity and that the actual spectrum is slightly flatter. Other differences between both models is that the spectral index curve of the combined data (bottom-left panel of Figure 14) seem to be more variable compared with the smoother spectral index curve in Figure 13, which is a consequence of the smaller derived decorrelation time. The residuals shown in the top-right panel of Figure 14 do not show evident trends, although there are several points above $5 \sigma$ which may indicate outliers. Additionally, the histogram of the residuals (bottom-right panel) of the combined data seems to be less consistent with a Gaussian distribution than that of Figure 13. It is possible that a different model, either with a more sophisticated spectral description of the data, or a better adapted stochastic process - like a CARMA or an infinite mixture model (Kelly et al. 2011, Takata et al. 2018) — could provide a better fit to the light-curves at all frequencies between Bands 3 and 10.

\subsection{Drawbacks and future refinements.}

To address the current limitations of the proposed modeling, first we must stress that the perspective of the current study is admittedly phenomenological. That is, it emphasizes the capability of the models to infer the behavior of the calibrators' fluxes rather than to understand the nature of their variability. The criterion which we use to evaluate whether a mixture of Ornstein-Uhlenbeck processes is an adequate model for the blazar variability is if it is capable of describing the light-curve variations leaving residuals consistent with Gaussian white noise at all frequencies.

On this vein, a more pertinent criticism may be our reliance on Gaussian distributions as generators of the stochasticity and noise. Indeed, the general theory of time series uses more general distributions (Brockwell \& Davis 2002), but in astronomy, 


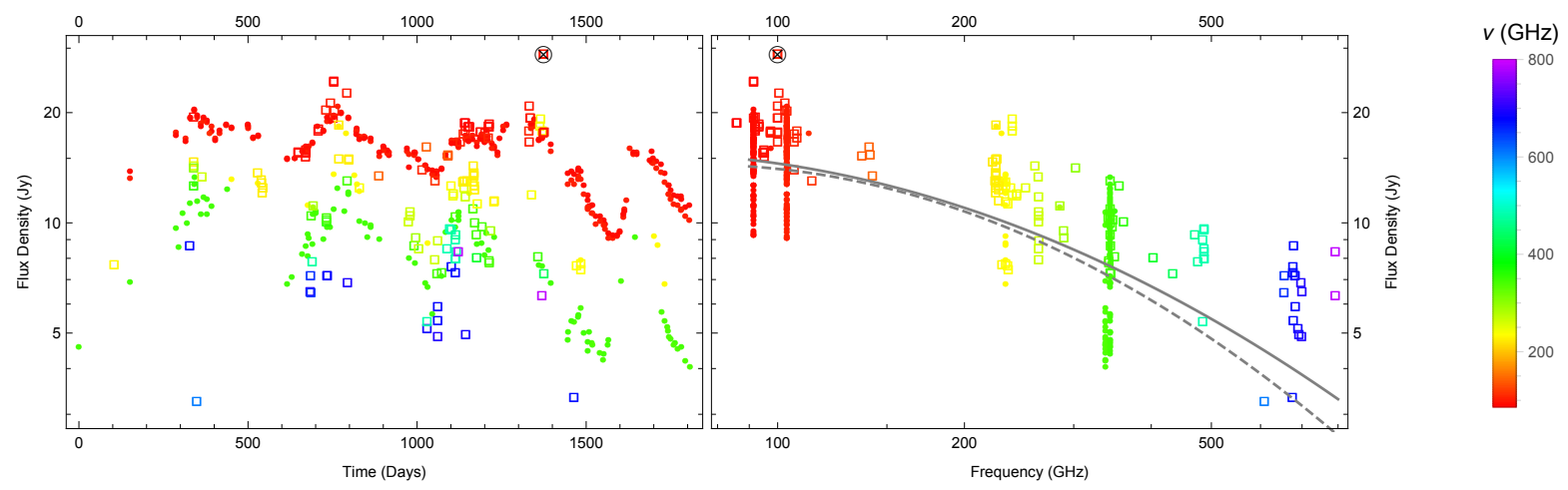

Figure 12. Combined data from the source catalog (filled circles) and from Bonato et al. (2018) (hollow squares) from J2253+1608. The $\otimes$ marks one masked outlier. Left panel: light-curve. Right panel: Spectrum. Grey continuous and dashed curves show the long-term mean spectrum fitted to the combined dataset and to only the source catalog data, respectively.
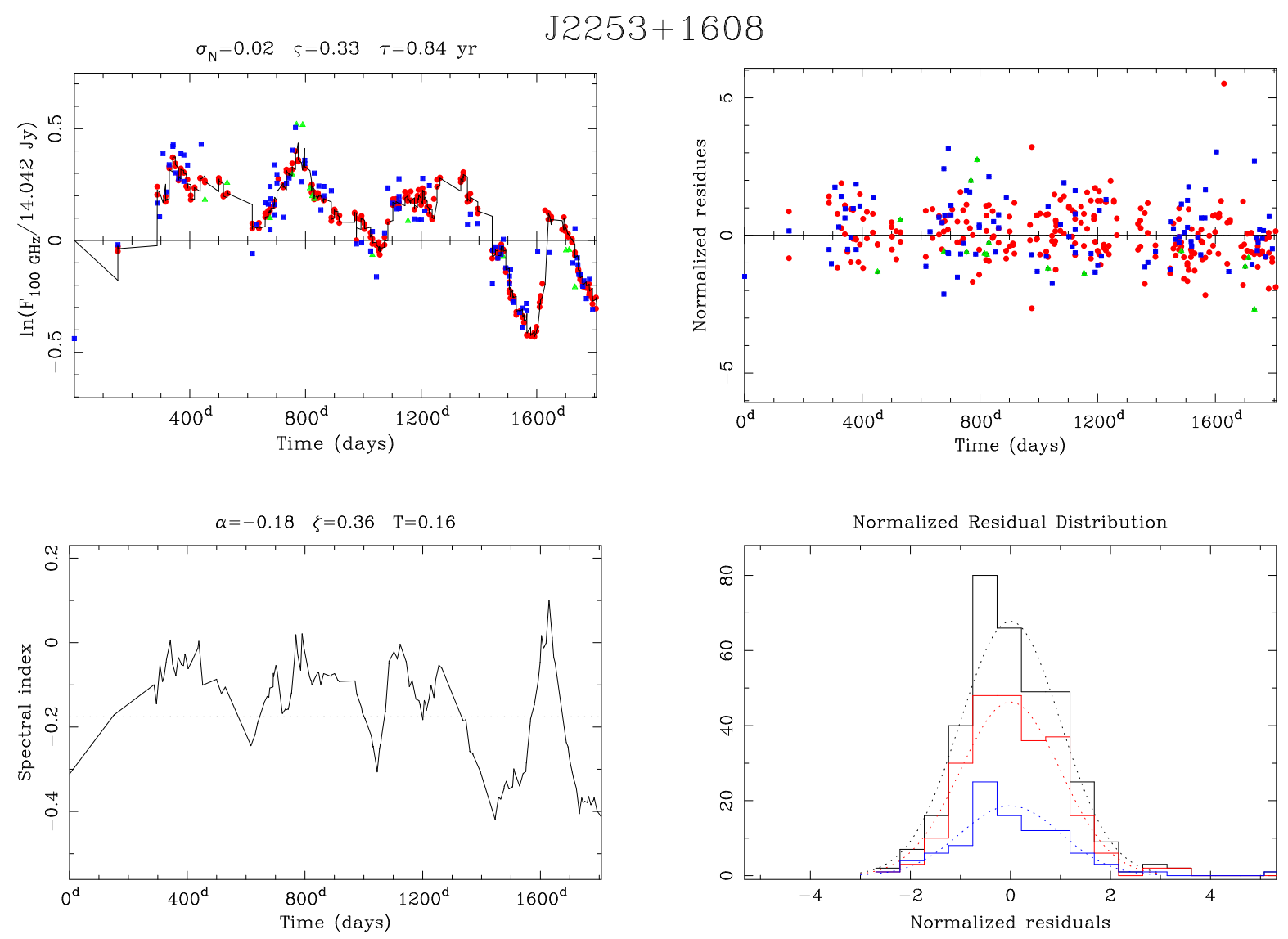

Figure 13. OU1F1a model with lag fitted to the source catalog data for J2253+1608. Panel arrangements, plotted quantities, and line colors follow the same convention as of Figure 8. 

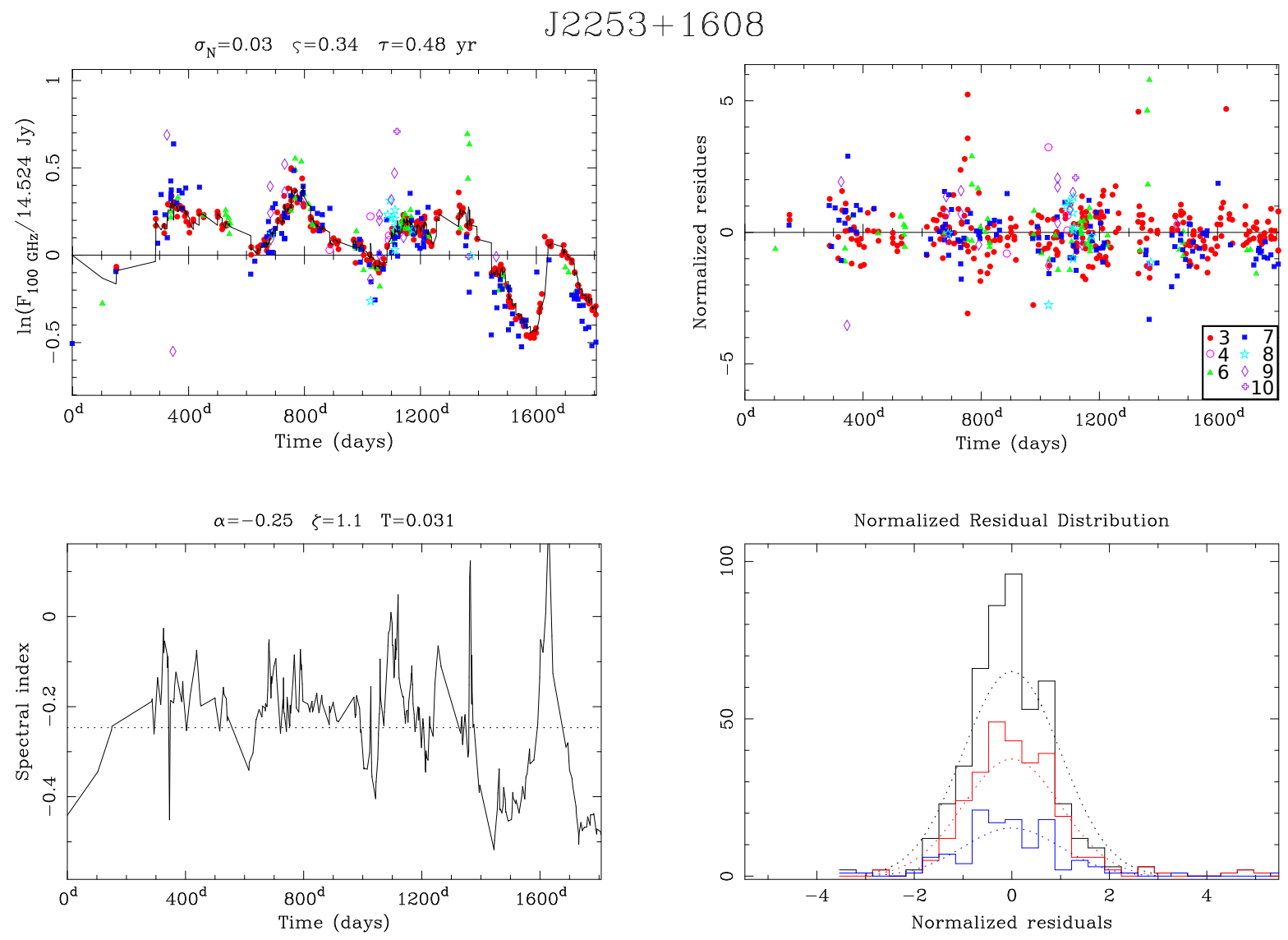

Figure 14. OU1F1a model with lag fitted to the combined data set (source catalog and Bonato et al. (2018)) for J2253+1608. Panel arrangements, plotted quantities, and line colors follow the same convention as of Figure 8. Top panels include measurements at Bands 3, 4, 6, 7, 8, 9, and 10, whose symbols are indicated in the legend of the top-right panel.

by far the majority of past and current development is in Gaussian time series modeling (e.g., Foreman-Mackey et al. 2017). Albeit it is common when stochastic methods are presented to remark that Gaussian hypotheses are not crucial, to explicitly present and explore a non-Gaussian model is rare (e.g., as done briefly in Eyheramendy et al. 2018). In any case, the present work is thought as a first step toward a more adequate calibrator modeling, which may not be based on Gaussian hypotheses.

Another limitations of our modeling are related with the current cadency of the calibrator survey and the limited time span of the monitoring. One direct consequence of the former is that the short-term (intraday) variability likely forms part of what we call white noise residuals $\left(\sigma_{N}\right)$, together with the instrumental and atmospheric noise. A dedicated study of this time-frequency domain (for example, by doing a high-cadency monitoring for a limited period of time) could break down which fraction of this noise is actually intrinsic to each source. Limitations stemming from short time span of the monitoring are of two types: unaccounted transient variability and systematic uncertainties and bias in the estimated parameters. The first one, more than 
a limitation, is the risk that the source just may unexpectedly change qualitatively the behavior it has characterized it during the last years. Longer studies of blazar variability may illustrate what the grid calibrators could do in the future in decade-long timescales. For example, monitoring of four grid calibrators in $\mathrm{a} \approx 14 \mathrm{yr}$ timescale was performed by the IRAM $30 \mathrm{~m}$ telescope in the $\mathrm{mm} / \mathrm{sub}-\mathrm{mm}$ frequencies (Trippe et al. 2011). These are J1229+0203, J1642+3948, J2253+1608, and J0319+4130; respectively, 3C 273, 3C 345, 3C 454.3, and 3C 84. The fluxes and spectral indices presented in Section 2 are consistent with those observations and the variations observed for the most part. However, the stochastic processes presented here will likely not be able to recover or forecast all variability features. Indeed, the change experienced by J2253+1608 in 2005 CE (Trippe et al. 2011, Fig. 1) is unlikely to be well described by a stationary stochastic process. More complete models for the future monitoring will likely need to include some non-stationarity, either in the deterministic model or in its stochastic parameters.

Alternatively, for some applications it may be desirable to select only a fraction (the most recent, for example) of the history of the light-curve. For example, in Figure 8 the top-right panel shows a somewhat larger scattering of residuals before $t=800$ d. Including the data from this more unstable phase of J0635-7516 in the modeling produces an increase of the uncertainty due to variability in the current forecasts. Indeed, removing these data from the modeling decreases the uncertainties associated to the forecasts. While this type of censoring of the data may be justified in some cases, we must bear in mind that a new phase of increased instability is essentially unpredictable. In a way, a larger uncertainty in the forecasts is the way the model accounts for the possible occurrence of another unstable phase in the future. Alternative models which include variation of more parameters with frequency (like the variance rates) or which include non-stationary flaring activity could provide more flexibility and allow for tighter uncertainty bounds for the forecast through the entire spectral coverage.

The second drawback derived from a relatively short monitoring time baseline is bias and systematic uncertainty of the fitted parameters. Specifically, the decorrelation time - which is defining of the OU-process - cannot be recovered. This is well illustrated by Kozłowski (2017b) and by Equation (A.6) in the case of an AR(1) process. From the latter equation is not difficult to see, for example, that the MLE of $\phi=\exp (-\delta t / \tau)$ is not located farther from 1 (where $\tau$ becomes unconstrained) than $\sqrt{\Delta T / 2 \tau}$ times the standard deviation of the estimator, regardless of the cadency or the number of samples $N$. Since the MLE is the asymptotically most efficient estimator, there is no really a way to improve the estimation of $\tau$ without increasing $\Delta T$, the total monitoring time. Kozłowski (2017b) illustrates this limitation by noting that a short time baseline cannot probe the short frequency section of the power spectrum of the process. Other non-parametric estimators of the decorrelation timescale (Koen 2012, Kozłowski 2017a) which may perform better for finite samples than the MLE are based on determining an accurate power spectra of the process, for which we require a more regular sampling. Note also that when $\Delta T / \tau$ is not sufficiently large, the long-term mean $\mu$ also becomes unconstrained. Roughly, $\Delta T / \tau$ gives the number of independent samples of the process. 
These are useful to determine $\mu$, whose MLE uncertainty goes like $\sqrt{\tau / 2 \Delta T}$ (A.4).

Fortunately, as shown in Sections 3.3 and 4.2, the large parametric uncertainty derived from a long decorrelation time does not produce a large uncertainty in the forecasts of the process, at least in the relatively short times characterizing the current cadency of the calibrator survey. In the long-term, the largest effect is produced by the uncertainty of $\mu$. When $\Delta T / \tau<10$, a procedure to determine the maximum parametric error we may incur due to the unconstrained $\tau$ may be to model the stochastic process with a regular (i.e., undamped) Brownian motion. Alternatively, resampling the likelihood with the bayesian procedure presented in Section 4.2 can provide in principle a better estimation for the parametric uncertainty when $\tau$ is less constrained.

\section{Conclusions}

We present a stochastic modeling of the time series measurements made by ALMA of a list of 39 blazar sources used as flux calibrators. The main results, conclusions, and advantages of the procedures presented in this work are summarized as follows:

(i) Mixtures of Ornstein-Uhlenbeck (OU) process in flux and spectral index are able to model reasonably well the multi-frequency light-curves of the calibrators in the 90 $350 \mathrm{GHz}$ range, on timescales of $\sim 5 \mathrm{yr}$ and cadences of one measurement every 1-2 weeks. A single OU-process and a single spectral index provides a first model approximation. Further features of the modeling include additional stochastic process for the spectral index variations, additional curvature of the spectrum, and a frequency dependent time lag.

(ii) Using maximum likelihood estimation and based on a statistical assessment of the residuals, we determine that most (38) sources are better modeled with an additional OU-process describing the spectral index variations. In addition, 37 of the 39 sources evidence a decreasing spectral index with frequency. Five sources are apparently characterized by a significant frequency dependent lag between their Band 3 and 7 light-curves. Four of them are better described by a $\sim 2 \mathrm{~d}$ lag with high-frequency leading, and one source with a $7 \mathrm{~d}$ low-frequency leading lag.

(iii) The modeling provides forecasts and flux interpolations based on the history of the time-variable source, together with their associated uncertainties. These can be use to perform diagnostics on new measurements of the calibrators, determine flux uncertainties due to the variability of the secondary flux calibrator, and to evaluate the confidence of the flux calibration in the ensuing data quality assurance.

(iv) Ill-constrained decorrelation times of the OU-process due to monitoring limitations should not greatly affect the determination of forecasts and interpolations. These are the most relevant quantities for the practical use in the ALMA calibration routine.

(v) Additional advantages of the formalism presented in this work are the flexibility they accommodate inhomogeneous sampling, updated measurements, and potentially higher frequency data. Future improvements could include 
further refinements in the noise terms (e.g., separating explicitly atmospheric and instrumental) and inclusion of non-Gaussian hypotheses.

\section{Acknowledgments}

Authors thank M. Bonato for helping and answering our questions about ALMACAL. Authors also thank an anonymous referee for detailed comments which have improved this paper. This paper makes use of ALMA data: ADS/JAO.ALMA\#2011.0.00001.CAL. ALMA is a partnership of ESO (representing its member states), NSF (USA) and NINS (Japan), together with NRC (Canada), MOST and ASIAA (Taiwan), and KASI (Republic of Korea), in cooperation with the Republic of Chile. The Joint ALMA Observatory is operated by ESO, AUI/NRAO and NAOJ. In addition to already $\mathrm{R}$ cited packages, this publication made use of stringr (Wickham 2018) and forecast (Hyndman et al. 2018). Analysis presented here made use of the Perl Data Language (PDL, http://pdl.perl.org) developed by K. Glazebrook, J. Brinchmann, J. Cerney, C. DeForest, D. Hunt, T. Jenness, T. Lukka, R. Schwebel, and C. Soeller.

\section{Appendix A. Homogeneous sampling without noise: the AR (1) model.}

The AR(1) process is one of the simplest processes which fulfills the features described in section 2 : is characterized by a long-term mean $(\mu)$ with a correlation between terms which decreases monotonically with time-lag. The Gaussian AR(1) process is defined by the following recurrence

$$
x_{n}-\mu=\phi\left(x_{n-1}-\mu\right)+\sigma^{2} Z_{n},
$$

where the $Z_{n}$ is a standard GWN and $0<\phi<1$. This process is Markovian, that is, the distribution of $x_{n}$ given the entire series until the $(n-1)$-th term $\left(\ldots, x_{0}, x_{1}, \ldots, x_{n-1}\right)$ depends solely on $x_{n-1}$. Sometimes the process receives the name "mean-reverting" because $x_{n}$ is distributed around a value closer to $\mu$ than $x_{n-1}$. Defined in this way, the covariance between two terms is given by

$$
\operatorname{cov}\left(\mathrm{x}_{\mathrm{n}+\mathrm{h}}, \mathrm{x}_{\mathrm{n}}\right)=\frac{\sigma^{2} \phi^{\mathrm{h}}}{1-\phi^{2}}
$$

that is, it depends only on the time difference (stationary process).

A simple interpretation may assign the $x_{n}$ to the log-flux of a calibrator measured at a time $n$. In this case, the time series correspond to equally spaced observations. As shown in (Appendix A), $\phi$ modulates the degree of correlation between consecutive measurements and thus it must depend on the time between observations. For $\phi \ll 1$, the $x_{n}$ are almost uncorrelated between each other, characteristic of largely spaced observations. Analogously, $\phi \approx 1$ could represent tightly correlated, very frequent measurements. Indeed, the $\mathrm{AR}(1)$ series can be interpreted as the discrete, homogeneous sampling of an OU continuous time process. This process also receives the name continuous AR process (CAR) and damped random walk. Under this interpretation,

$$
\phi=e^{-\delta t / \tau},
$$


where $\tau$ is known as the decorrelation timescale, and $\delta t$ is the time between consecutive samplings. It is worth noticing that because the time-sampling is homogeneous, the discrete series is stationary. If the time sampling where inhomogeneous, then $\delta t$ and $\phi$ would depend on the index of the series. Thus, the process would no longer be stationary, but only due to the sampling: the continuous process is still stationary.

The parameters of an $\operatorname{AR}(1)$ process $\left(\mu, \phi, \sigma^{2}\right)$ can be estimated from a specific sample (or realization) of the process, $\operatorname{say}\left\{x_{n}\right\}_{n=1}^{N}$. Under reasonably general circumstances (Eyheramendy et al. 2018), the irregular process is ergodic for the mean, and $\mu$ can be approximated efficiently by the average of the $x_{n}$. In the $\operatorname{AR}(1)$ case, the average distributes approximately normal with

$$
\hat{\mu}=\frac{1}{N} \sum_{n=1}^{N} x_{n} \approx \mathcal{N}\left(\mu, \frac{\sigma^{2}}{N(1-\phi)^{2}}\right) .
$$

We see immediately that for $\phi \approx 1-$ or following (A.3), $\delta t \ll \tau-$ the estimations of the long term mean becomes more uncertain.

We define the centered process $\tilde{x}_{n}=x_{n}-\mu$, which is an $\operatorname{AR}(1)$ process with zero long-term mean. In practice, we approximate $\tilde{x}_{n}$ using $x_{n}-\hat{\mu}$. Parameters $\phi$ and $\sigma^{2}$ can be estimated using maximum likelihood on $\tilde{x}_{n}$, assuming it is an $\mathrm{AR}(1)$ process. The log-likelihood function is given by

$$
l\left(\phi, \sigma^{2}\right)=-\frac{N}{2} \ln \left(2 \pi \sigma^{2}\right)-\frac{1}{2 \sigma^{2}} \sum_{n=1}^{N}\left(\tilde{x}_{n}-\phi \tilde{x}_{n-1}\right)^{2},
$$

assuming $\tilde{x}_{0}=0$. This last requirement is artificial, but not relevant for large $N$ (Hamilton 1994). The maximum likelihood estimators (MLE) and its normal asymptotic distributions are given by

$$
\begin{aligned}
& \hat{\phi}=\frac{\sum_{n=1}^{N} \tilde{x}_{n-1} \tilde{x}_{n}}{\sum_{n=1}^{N} \tilde{x}_{n-1}^{2}} \approx \mathcal{N}\left(\phi, \frac{1-\phi^{2}}{N}\right), \\
& \hat{\sigma^{2}}=\frac{1}{N} \sum_{n=1}^{N}\left(\tilde{x}_{n}-\hat{\phi} \tilde{x}_{n-1}\right)^{2} \approx \mathcal{N}\left(\sigma^{2}, \frac{2 \sigma^{2}}{N}\right) .
\end{aligned}
$$

It is also possible to give simple formulae for the best - in the sense of minimum mean square error - predictions and interpolations. The best prediction (or forecast) of the $(n+h)$-th term of a centered $\mathrm{AR}(1)$ process given the $(n-1)$ previous observations $\left(\tilde{x}_{n+h \mid n-1}, h \geq 0\right)$ is given by (Brockwell \& Davis 2002)

$$
\tilde{x}_{n+h \mid n-1}=\phi^{h+1} \tilde{x}_{n-1}, \quad \mathrm{E}\left(\tilde{x}_{n+h}-\tilde{x}_{n+h \mid n-1}\right)^{2}=\frac{1-\phi^{2 h+2}}{1-\phi^{2}} \sigma^{2},
$$

where the second equation gives the mean square error of the prediction. Similarly, based on observations of $\tilde{x}_{i}, i=1, \ldots(n-1)$ and $\tilde{x}_{n+k}$, the best interpolation for $\tilde{x}_{n+h}$ $(0 \leq h<k)$ is

$$
\mathrm{E}\left(\tilde{x}_{n+h} \mid \tilde{x}_{1}, \ldots \tilde{x}_{n-1}, \tilde{x}_{n+k}\right)=\frac{\tilde{x}_{n-1} \phi^{h+1}\left(1-\phi^{2(k-h)}\right)+\tilde{x}_{n+k} \phi^{k-h}\left(1-\phi^{2(h+1)}\right)}{1-\phi^{2(k+1)}} .
$$


Note that (A.9) depends only on the measurements taken immediately after and before time $n$. When $\phi \rightarrow 1$, (A.9) becomes the linear interpolation between $\tilde{x}_{n-1}$ and $\tilde{x}_{n+k}$. The mean squared error is

$$
\mathrm{E}\left(\tilde{x}_{n+h}-\mathrm{E}\left(\tilde{x}_{n+h} \mid \tilde{x}_{n-1}, \tilde{x}_{n+k}\right)\right)^{2}=\sigma^{2} \frac{1-\phi^{2(h+1)}}{1-\phi^{2}} \frac{1-\phi^{2(k-h)}}{1-\phi^{2(k+1)}} .
$$

This last expression tends to the prediction error (A.8) when $k$ is large. As can be expected, observations in the distant past or future are only useful to determine the global parameters of the process, but their specific values do not constrain estimations or forecasts at present time.

We can estimate the influence of the uncertainty of the typically unknown parameter $\mu$ by assuming we replaced it with a random variable $\hat{\mu}$ with mean $\mu$ and variance $\sigma_{\mu}^{2}$. According to (A.8), the best predictor of $x_{n+h}$ given $n-1$ observations $\left(x_{n+h \mid n-1}\right)$ would be $\hat{\mu}+\phi^{h+1}\left(x_{n-1}-\hat{\mu}\right)$. Therefore, the variance of $x_{n+h \mid n-1}$ due to the $\mu$-uncertainty that is, the parametric uncertainty - and to the stochastic variability are, respectively,

$$
\left(1-\phi^{h+1}\right)^{2} \sigma_{\mu}^{2}, \quad \sigma^{2} \frac{1-\phi^{2 h+2}}{1-\phi^{2}} \leq \sigma^{2}(h+1) .
$$

A short time between the last observation and the prediction is characterized by low values of $h$ and $\phi \approx 1$, which decreases the uncertainty due to the unknown $\mu$. Note that we expect a low influence of $\mu$ in the prediction uncertainty also if $\tau$ is very large. In this latter case, the process becomes closer to a Brownian motion and the concept of long-term mean is meaningless.

\section{Appendix B. State space representation and Kalman recursions}

We follow the notation and treatment of Kitagawa \& Gersch (1996). A time series $Y_{n}$ allows a Gaussian state space representation if it can be expressed in the following way

$$
\begin{aligned}
& Y_{n}=H_{n} X_{n}+\epsilon_{n}, \\
& X_{n+1}=F_{n} X_{n}+G_{n} w_{n} \\
& \left(\begin{array}{c}
\epsilon_{n} \\
w_{n}
\end{array}\right) \sim \mathcal{N}\left(\mathbf{0},\left[\begin{array}{cc}
R_{n} & 0 \\
0 & Q_{n}
\end{array}\right]\right),
\end{aligned}
$$

where (B.1) and (B.2) are called the measurement and state equations, respectively. The random variables $\epsilon_{n}$ and $w_{n}$ are uncorrelated. In the case of an OU-rFsa process, (B.1) corresponds to (8) with

$$
H_{n}=(\underbrace{1, \ldots, 1}_{r}, \underbrace{\ln \left(\nu_{n} / \nu_{0}\right), \ldots, \ln \left(\nu_{n} / \nu_{0}\right)}_{s}), \quad R_{n}=\sigma_{N}^{2}\left(\nu_{n}\right) .
$$

Equation (B.2) corresponds to (9) with $F_{n}=\exp \left(-\delta t_{n} \boldsymbol{\tau}^{-1}\right)$ and $X_{n}$ being the vector in the left hand of (9). Unless one intends to simulate the process, it is not necessary to define $G_{n}$ and $Q_{n}$ explicitly but only up to the requirement that $G_{n} Q_{n} G_{n}^{\mathrm{T}}=\operatorname{cov}\left(\eta_{\mathrm{n}}\right)$ (see (12)). We denote in this Appendix a general process as $Y_{n}$. A lowercase $y_{n}$ refers specifically to the stationary model adjusted to a calibrator, as in (3). 
Let us denote $X_{n \mid n-1}$ the conditional expectation of $X_{n}$ given all the previous information, which is represented by $\left(Y_{1}, \ldots, Y_{n-1}\right), i=1, \ldots, n-1$. We define $V_{n \mid n-1}=\mathrm{E}\left(X_{n}-X_{n \mid n-1}\right)\left(X_{n}-X_{n \mid n-1}\right)^{\mathrm{T}}$, that is, the variance of the predictor. The $Y_{i}$ represent either an observation of $Y(t)$ at time $t_{i}$, or a filler value in case no observation was taken at time $t_{i}$. This flexibility will be useful later to introduce best predictions at time $t_{i}$ given all the other observations (the interpolation or smoothing problem). Evidently, this filler value should have no influence in the calculated best estimators. We define respectively $X_{1 \mid 0}$ and $V_{1 \mid 0}$ as the predictor and variance of $X_{1}$, assuming no previous information. It is useful sometime consider that a "previous" observation of the process $Y_{0}$ took place in the infinite past $\left(\delta t_{1}=t_{1}-t_{0} \rightarrow+\infty\right)$, and is therefore uninformative. Analogously, let $X_{n \mid n}$ and $V_{n \mid n}$ denote the conditional expectation of $X_{n}$ and its variance, respectively, given information $Y_{i}$ up to $i=n$.

The first set of Kalman recursions are

$$
\begin{aligned}
& K_{n}=\left\{\begin{array}{ll}
V_{n \mid n-1} H_{n}^{\mathrm{T}}\left(H_{n} V_{n \mid n-1} H_{n}^{\mathrm{T}}+R_{n}\right)^{-1} & ; Y_{n} \text { observed } \\
0 & ; Y_{n} \text { not observed }
\end{array},\right. \\
& X_{n \mid n}=X_{n \mid n-1}+K_{n}\left(Y_{n}-H_{n} X_{n \mid n-1}\right), \\
& V_{n \mid n}=\left(I-K_{n} H_{n}\right) V_{n \mid n-1}, \\
& X_{n+1 \mid n}=F_{n+1} X_{n \mid n} \\
& V_{n+1 \mid n}=F_{n+1} V_{n \mid n} F_{n+1}^{\mathrm{T}}+G_{n+1} Q_{n+1} G_{n+1}^{\mathrm{T}},
\end{aligned}
$$

for $n \geq 1$. Equations (B.5) to (B.7) define the Kalman filter, and Equations (B.8) and (B.9) define the Kalman predictions. $K_{n}$ is known as the Kalman gain. The least mean square prediction for $Y_{n}$ and its variance are

$Y_{n \mid n-1}=H_{n} X_{n \mid n-1} \quad, \quad \mathrm{E}\left(Y_{n}-Y_{n \mid n-1}\right)^{2}=: r_{n}=H_{n} V_{n \mid n-1} H_{n}^{\mathrm{T}}+R_{n}$.

The Kalman predictions are useful not only as forecast, but also to calculate the log-likelihood, which is given by

$$
-2 \ln \mathcal{L}=\sum_{n \text { observed }} \ln \left(2 \pi r_{n}\right)+\frac{\left(Y_{n}-Y_{n \mid n-1}\right)^{2}}{r_{n}} .
$$

The second set of Kalman recursions are

$$
\begin{aligned}
& A_{n}=V_{n \mid n} F_{n+1}^{\mathrm{T}} V_{n+1 \mid n}^{-1}, \\
& X_{n \mid N}=X_{n \mid n}+A_{n}\left(X_{n+1 \mid N}-X_{n+1 \mid N}\right), \\
& V_{n \mid N}=V_{n \mid n}+A_{n}\left(V_{n+1 \mid N}-V_{n+1 \mid n}\right) A_{n}^{\mathrm{T}},
\end{aligned}
$$

where $N>n$. In (B.12), $V_{n+1 \mid n}^{-1}$ can be replaced by a pseudo-inverse in case is not invertible (Anderson \& Moore 2012). Equations (B.12)-(B.14) define the Kalman smoothing, and give the best prediction of $X_{n}$ given all the $Y_{i}$ (past and future) until $N$. The interpolated expected value of $Y(t)$ at $t_{n}$ (assuming it was not observed), given all the data, is $Y_{n \mid N}=H_{n} X_{n \mid N}$. 


\section{Appendix C. Supplementary material}

In the supplementary material we provide additional results, plots, and $\mathrm{R}$ (v. 3.5.0) computer programs to generate the simulated datasets presented through the paper. The supplementary material includes plots like those of Figures 1, 6, 7 and 8 for each source, and for all models specified in Table 3. A log file for each model gives details about the MLE, including the final error and cross-correlation matrices, AIC, $\mathrm{p}_{\mathrm{A}-\mathrm{D}}$, and

pL-B values. A text file also indicates the 20 measurements masked from the catalog as possible outliers. We also include data and details of the modeling for the non-grid calibrators analyzed in Section 5.1. As indicated in the text, we provide the scripts used to generate the synthetic light-curves used in Section 3.4. PDL code implementing the Kalman recursions (Appendix A) is given in the supplementary material and can also be obtained from the Astrophysics Source Code Library (Guzmán 2019).

\section{References}

Anderson B \& Moore J 2012 Optimal Filtering Dover Books on Electrical Engineering Dover Publications.

Andrae R, Kim D W \& Bailer-Jones C A L 2013 A\&A 554, A137.

Begelman M C, Blandford R D \& Rees M J 1984 Reviews of Modern Physics 56, 255-351.

Bevington P R \& Robinson D K 2003 Data reduction and error analysis for the physical sciences McGraw-Hill.

Blandford R D \& Königl A 1979 ApJ 232, 34-48.

Bonato M, Liuzzo E, Giannetti A, Massardi M, De Zotti G, Burkutean S, Galluzzi V, Negrello M, Baronchelli I, Brand J, Zwaan M A, Rygl K L J, Marchili N, Klitsch A \& Oteo I 2018 MNRAS 478, 1512-1519.

Brockwell P J \& Davis R A 2002 Introduction to Time Series and Forecasting Springer-Verlag New York.

Butler B 2012 Flux Density Models for Solar System Bodies in CASA. ALMA Memo \# 594.

URL: https://science.nrao.edu/facilities/alma/aboutALMA/Technology/ALMA_Memo_Series/alma594/abs594

Edelson R, Mushotzky R, Vaughan S, Scargle J, Gandhi P, Malkan M \& Baumgartner W 2013 ApJ 766, 16.

Eyheramendy S, Elorrieta F \& Palma W 2018 MNRAS 481, 4311-4322.

Falomo R, Pian E \& Treves A 2014 Astronomy and Astrophysics Review 22, 73.

Faraway J, Marsaglia G, Marsaglia J \& Baddeley A 2017 goftest: Classical Goodness-of-Fit Tests for Univariate Distributions. R package version 1.1-1.

URL: https://CRAN.R-project.org/package=goftest

Feigelson E \& Babu G 2012 Modern Statistical Methods for Astronomy: With R Applications Cambridge University Press, Cambridge.

Feigelson E D, Babu G J \& Caceres G A 2018 Frontiers in Physics 6, 80.

Fomalont E, van Kempen T, Kneissl R, Marcelino N, Barkats D, Corder S, Cortes P, Hills R, Lucas R, Manning A \& Peck A 2014 The Messenger 155, 19-22.

Foreman-Mackey D, Agol E, Ambikasaran S \& Angus R 2017 AJ 154, 220.

Fromm C M, Fuhrmann L \& Perucho M 2015 A\&A 580, A94.

Fuhrmann L, Larsson S, Chiang J, Angelakis E, Zensus J A, Nestoras I, Krichbaum T Â P, Ungerechts H, Sievers A, Pavlidou V, Readhead A C S, Max-Moerbeck W \& Pearson T J 2014 MNRAS 441, 1899-1909.

Goyal A, Stawarz E, Zola S, Marchenko V, Soida M, Nilsson K, Ciprini S, Baran A, Ostrowski M, 
Wiita P J, Gopal-Krishna, Siemiginowska A, Sobolewska M, Jorstad S, Marscher A, Aller M F, Aller H D, Hovatta T, Caton D B, Reichart D, Matsumoto K, Sadakane K, Gazeas K, Kidger M, Piirola V, Jermak H, Alicavus F, Baliyan K S, Baransky A, Berdyugin A, Blay P, Boumis P, Boyd D, Bufan Y, Campas Torrent M, Campos F, Carrillo Gómez J, Dalessio J, Debski B, Dimitrov D, Drozdz M, Er H, Erdem A, Escartin Pérez A, Fallah Ramazani V, Filippenko A V, Gafton E, Garcia F, Godunova V, Gómez Pinilla F, Gopinathan M, Haislip J B, Haque S, Harmanen J, Hudec R, Hurst G, Ivarsen K M, Joshi A, Kagitani M, Karaman N, Karjalainen R, Kaur N, Kozieł-Wierzbowska D, Kuligowska E, Kundera T, Kurowski S, Kvammen A, LaCluyze A P, Lee B C, Liakos A, Lozano de Haro J, Moore J P, Mugrauer M, Naves Nogues R, Neely A W, Ogloza W, Okano S, Pajdosz U, Pandey J C, Perri M, Poyner G, Provencal J, Pursimo T, Raj A, Rajkumar B, Reinthal R, Reynolds T, Saario J, Sadegi S, Sakanoi T, Salto González J L, Sameer, Simon A O, Siwak M, Schweyer T, Soldán Alfaro F C, Sonbas E, Strobl J, Takalo L O, Tremosa Espasa L, Valdes J R, Vasylenko V V, Verrecchia F, Webb J R, Yoneda M, Zejmo M, Zheng W, Zielinski P, Janik J, Chavushyan V, Mohammed I, Cheung C C \& Giroletti M 2018 ApJ 863, 175.

Granger C W J \& Morris M J 1976 Journal of the Royal Statistical Society. Series A (General) 139(2), 246-257.

URL: http://www.jstor.org/stable/2345178

Gregory P 2005 Bayesian Logical Data Analysis for the Physical Sciences: A Comparative Approach

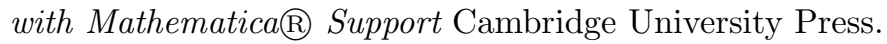

URL: https://books.google.co.jp/books?id=yJ_5VFoOzGMC

Guzmán A E 2019 'Kalman: Forecasts and interpolations for ALMA calibrator variability' Astrophysics Source Code Library.

URL: https://ascl.net/1906.005

Hada K, Doi A, Kino M, Nagai H, Hagiwara Y \& Kawaguchi N 2011 Nature 477, 185-187.

Hamilton J 1994 Time Series Analysis Princeton University Press.

Hyndman R, Athanasopoulos G, Bergmeir C, Caceres G, Chhay L, O'Hara-Wild M, Petropoulos F, Razbash S, Wang E \& Yasmeen F 2018 forecast: Forecasting functions for time series and linear models. R package version 8.4.

URL: http://pkg.robjhyndman.com/forecast

James F \& Roos M 1975 Computer Physics Communications 10(6), 343 - 367.

URL: http://www.sciencedirect.com/science/article/pii/0010465575900399

Joshi M \& Böttcher M 2011 ApJ 727, 21.

Kasliwal V P, Vogeley M S \& Richards G T 2015 MNRAS 451(4), 4328-4345.

Kellermann K I \& Verschuur G L 1988 Galactic and extragalactic radio astronomy (2nd edition) Springer-Verlag, Berlin and New York).

Kelly B C, Bechtold J \& Siemiginowska A 2009 ApJ 698, 895-910.

Kelly B C, Becker A C, Sobolewska M, Siemiginowska A \& Uttley P 2014 ApJ 788, 33.

Kelly B C, Sobolewska M \& Siemiginowska A 2011 ApJ 730, 52.

Kitagawa G \& Gersch W 1996 Smoothness Priors Analysis of Time Series Ima Volumes in Mathematics and Its Applications Springer Science \& Business Media, New York.

Koen C 2005 MNRAS 361, 887-896.

Koen C 2012 MNRAS 419, 1197-1218.

Kozłowski S 2017a ApJ 835, 250.

Kozłowski S 2017b A\&A 597, A128.

Kozłowski S, Kochanek C S, Udalski A, Wyrzykowski Ł, Soszyński I, Szymański M K, Kubiak M, Pietrzyński G, Szewczyk O, Ulaczyk K, Poleski R \& OGLE Collaboration 2010 ApJ 708(2), 927945.

Kushwaha P, Chandra S, Misra R, Sahayanathan S, Singh K P \& Baliyan K S 2016 ApJ 822, L13.

Liodakis I, Pavlidou V, Hovatta T, Max-Moerbeck W, Pearson T J, Richards J L \& Readhead A C S 2017 MNRAS 467, 4565-4576. 
MacLeod C L, Ivezić Ž, Kochanek C S, Kozłowski S, Kelly B, Bullock E, Kimball A, Sesar B, Westman D, Brooks K, Gibson R, Becker A C \& de Vries W H 2010 ApJ 721(2), 1014-1033.

Moreno R \& Guilloteau S 2002 An Amplitude Calibration Strategy for ALMA. ALMA Memo \# 372. URL: http://library.nrao.edu/public/memos/alma/main/memo372.pdf

Oteo I, Zwaan M A, Ivison R J, Smail I \& Biggs A D 2016 ApJ 822(1), 36.

R Core Team 2018 R: A Language and Environment for Statistical Computing R Foundation for Statistical Computing Vienna, Austria.

URL: https://www.R-project.org/

Ruan J J, Anderson S F, MacLeod C L, Becker A C, Burnett T H, Davenport J R A, Ivezić Ž, Kochanek C S, Plotkin R M, Sesar B \& Stuart J S 2012 ApJ 760, 51.

Scargle J D 1981 ApJS 45, 1-71.

Scargle J D 1982 ApJ 263, 835-853.

Sokolov A, Marscher A P \& McHardy I M 2004 ApJ 613, 725-746.

Takata T, Mukuta Y \& Mizumoto Y 2018 ApJ 869(2), 178.

Trippe S, Krips M, Piétu V, Neri R, Winters J M, Gueth F, Bremer M, Salome P, Moreno R, Boissier J \& Fontani F 2011 A\&A 533, A97.

Ulrich M H, Maraschi L \& Urry C M 1997 ARA\&A 35, 445-502.

van Kempen T A, Kneissl R, Marcelino N, Fomalont E B, Barkats S A, Corder S A, Lucas R, Peck A B \& Hills R 2014 The ALMA Calibrator Database I: Measurements taken during the commissioning phase of ALMA. ALMA Memo \# 599.

URL: http://library.nrao.edu/public/memos/alma/main/memo599.pdf

van Kempen T, Corder S, Lucas R \& Mauersberger R 2012 ALMA Newsletter 9, 8-16.

VanderPlas J T 2018 ApJS 236, 16.

Wagner S J \& Witzel A 1995 ARA\&A 33, 163-198.

Wang H \& Shi Y 2019 Astrophysics and Space Science 364(2), 27.

Warmels R, Biggs A, Cortes P, A., Dent B, Di Francesco J, Fomalont E, Hales A, Kameno S, Mason B, Philips N, Remijan A, Saini K, Stoehr F, Vila Vilaro B \& Villard E 2018 ALMA Technical Handbook, ALMA Doc. 6.3, ver. 1.0. ALMA Documents.

URL: $\quad$ https://almascience.nrao.edu/documents-and-tools/cycle6/alma-technicalhandbook/view

Wickham H 2018 stringr: Simple, Consistent Wrappers for Common String Operations. R package version 1.3.1.

URL: https://CRAN.R-project.org/package=stringr

Xue R, Luo D, Du L M, Wang Z R, Xie Z H, Yi T F, Xiong D R, Xu Y B, Liu W G \& Yu X L 2016 MNRAS 463, 3038-3055.

Yun M S, Mangum J, Bastian T \& Holdaway M 1998 Accurate Amplitude and Flux Calibration of the MMA. ALMA Memo \# 211.

URL: http://library.nrao.edu/public/memos/alma/main/memo211.pdf 